\title{
The synthesis of phosphonic acids derived from homocysteine via transesterification reactions
}

\author{
Jan Pícha, Miloš Buděšínský, Pavel Fiedler and Jiří Jiráček * \\ Institute of Organic Chemistry and Biochemistry, Academy of Sciences of the Czech Republic, \\ v.v.i., Flemingovo nám. 2, 16610 Prague 6, Czech Republic \\ E-mail:jiracek@uochb.cas.cz
}

Dedicated to Professor Pawel Kafarski to honor the achievements within his career

\begin{abstract}
An efficient methodology for the synthesis of S-substituted derivatives of phosphonohomocysteine has been developed. It starts from the corresponding thiols and is based on a synthetic sequence consisting of (i) the Kabachnik-Fields reaction, (ii) the transesterification of the diphenylphosphoryl moiety and (iii) the acidic deprotection of the phosphoryl and amino groups. The proposed synthetic procedures describing syntheses of phosphonohomocysteine precursors with di-tert-butylphosphoryl and tert-butyloxycarbonylamino groups could be useful for the preparation of novel $S$-substituted phosphonohomocysteine derivatives.
\end{abstract}

Keywords: Aminophosphonic acid, phosphonohomocysteine, protecting groups, acidolabile groups, transesterification

\section{Introduction}

In the course of our search for new inhibitors of betaine-homocysteine $S$-methyltransferases $\left(\mathrm{BHMT}^{1-3}\right.$ and BHMT $2^{4}$ ) we aimed to synthesize phosphonoanalogues of homocystine and homocysteine. Phosphonic ${ }^{5}$ and phosphinic $^{6}$ amino acids are well known inhibitors of different metalloenzymes.

The previously published preparation ${ }^{7}$ of phosphonohomocystine 3 (Scheme 1) consists of the synthesis of a phosphonohomocysteine intermediate bearing an acidolabile tert-butyl $S$ protecting group followed by the acidic hydrolysis of this group and a mild oxidation of the resulting free thiol group. The available literature ${ }^{7-9}$ offers several methods for the preparation of phosphonohomocysteine-derived analogues with different $S$-linked unbranched alkyl chains. However, there is no information concerning less stable $S$-linked groups. 
The aim of this study was to find the most suitable protecting groups for the phosphoryl and amino moieties of phosphonohomocysteine with regard to the lability of the RS- moiety under acidic conditions. This strategy could facilitate the preparation of phosphonohomocysteine derivatives with different alkyl or aryl substituents on the sulfur atom.

Therefore, we introduced three different hydrocarbon $S$-substituents ( $\mathrm{Ph}$, tert-butyl, $\mathrm{Bn}$ ) to create four series of differently protected precursors $2 \mathbf{a}-\mathbf{c}, 7 \mathbf{a}-\mathbf{c}, \mathbf{8 a - c}$ and $9 \mathbf{a}-\mathbf{c}$ and examined the stability of their respective C-S bonds under various conditions (Scheme 2).

Although at first glance the synthesis of this type of compounds appears trivial, it may be complicated because some important methods used for the synthesis aliphatic aminophosphonic acids are prohibited in the case of sulfur. For example, the use of the catalytic hydrogenation is excluded due to poisoning effect of sulfur, and sulfides tend to be oxidized by some agents to sulfoxides or sulfones.

Our synthetic approach is based on a three-step sequence. The first step consists of four variants of the three-component Kabachnik-Fields reaction: ${ }^{10}$ the condensation of (a) aldehydes, benzylcarbamate and triphenylphosphite in glacial acetic acid; ${ }^{11,12}$ (b) aldehydes, $N$-phenyl thiourea and triphenylphosphite in glacial acetic acid; $;^{7,13,14}$ (c) aldehydes, tert-butyl carbamate and triphenylphosphite in DCM in the presence of $\mathrm{TiCl}_{4},{ }^{15}$ or (d) aldehydes, benzylcarbamate and dimethyl phosphite in acetyl chloride. ${ }^{16}$ The second step involves transesterification of the reaction products using potassium tert-butanolate for (a) and (c) or with sodium methanolate for (b) and (d). The third and final synthetic step is the removal of the protecting groups of the phosphoryl and amino moieties.

\section{Results and Discussion}

Our initial effort was focused on the synthesis of phosphonic acids starting from aldehydes through 1-phenylthioureido phosphonate intermediates $2^{7-9}$ Thus, the Michael addition of thiophenol, tert-butylmercaptan and benzylmercaptan to acrolein afforded the corresponding aldehydes 1a-c in high yield, and the subsequent condensation with $N$-phenylthiourea and triphenylphosphine in glacial acetic acid gave phosphonate precursors 2a-c (Scheme 1).

Heating 2a in concentrated hydrochloric acid furnished the expected free 1aminophosphonic acid 10a. As stated in the Introduction, the reflux of analogue $\mathbf{2 b}$ bearing a tert-butyl moiety generated the crude phosphonohomocysteine, ${ }^{13}$ which, after oxidation with iodine, ${ }^{7}$ gave the required phosphonohomocystine 3. In contrast to Kudzin's and Stec's work, ${ }^{7}$ the attempt to prepare acid 10c from 2c under these harsh conditions led, in our hands, only to a mixture of unknown products. 


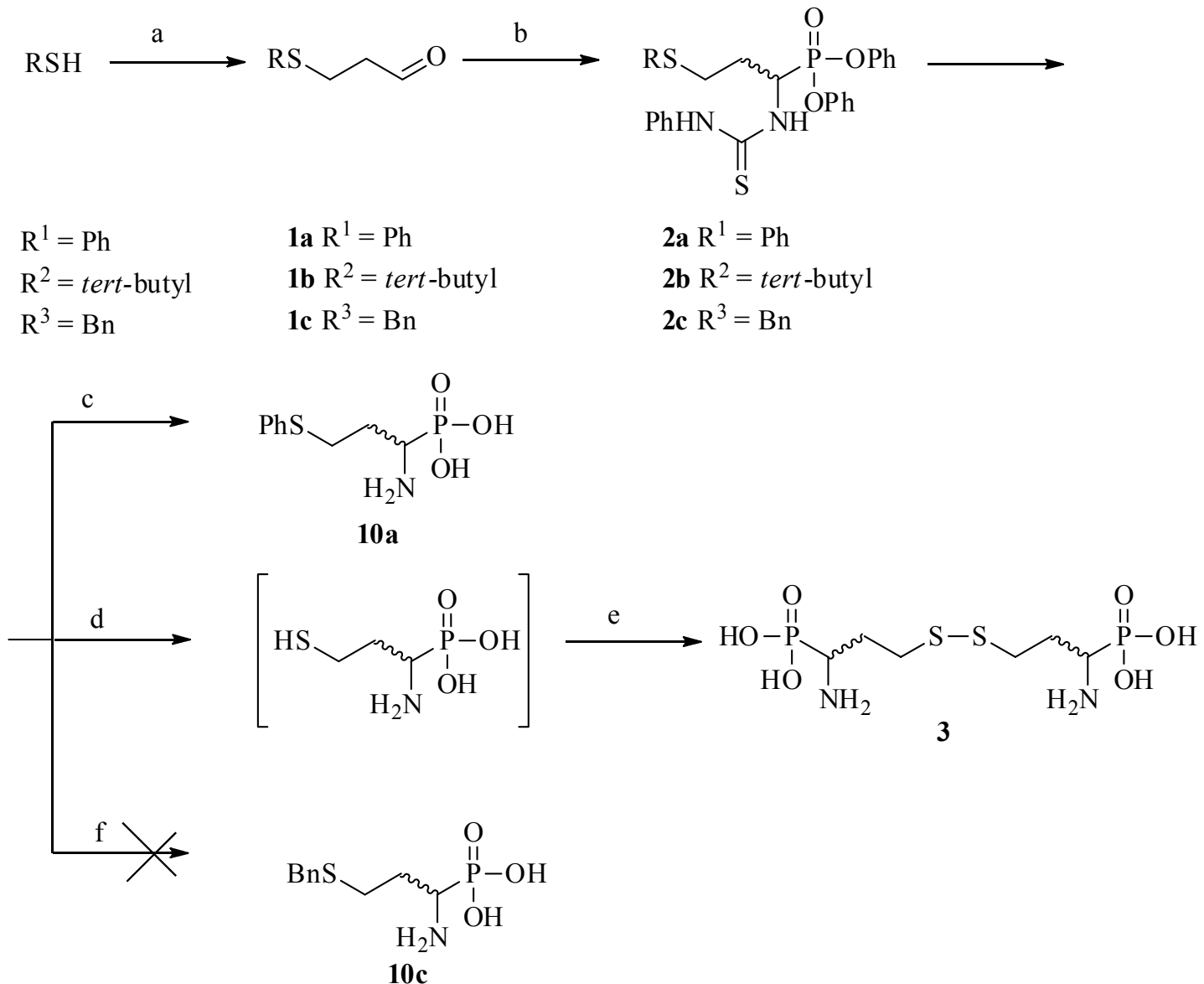

Scheme 1. Reactions, conditions and yields: (a) acrolein, TEA, dichloromethane, $0^{\circ} \mathrm{C}$ for $1 \mathrm{~h}$, then $\mathrm{rt}$ overnight, $\mathrm{R}^{1}=\mathrm{Ph}(79 \%)$ for $\mathbf{1 a}, \mathrm{R}^{2}=$ tert-butyl $(75 \%)$ for $\mathbf{1 b}, \mathrm{R}^{3}=\mathrm{Bn}(81 \%)$ for $\mathbf{1 c}$; (b) $N$-phenyl thiourea, triphenyl phosphite, $\mathrm{AcOH}, 80{ }^{\circ} \mathrm{C}, 1 \mathrm{~h}, \mathrm{R}^{1}=\mathrm{Ph}(54 \%)$ for $\mathbf{2 a}, \mathrm{R}^{2}=$ tert-butyl $(45 \%)$ for $\mathbf{2 b}, \mathrm{R}^{3}=\mathrm{Bn}(55 \%)$ for $\mathbf{2 c}$; (c) $35 \% \mathrm{HCl}$ and $\mathrm{AcOH}$, reflux for $12 \mathrm{~h}$, then 1,2epoxypropane, methanol, (67\%) starting from 2a; (d) 48\% $\mathrm{HBr}$ and $\mathrm{AcOH}$, reflux $14 \mathrm{~h}$; (e) $\mathrm{I}_{2}$, methanol and then 1,2-epoxypropane, methanol (65\% for two steps starting from $2 \mathbf{b}$ ); (f) starting from $2 \mathrm{c}$ under the same conditions as for (c).

The observed lability of the $\left(\mathrm{CH}_{3}\right)_{3} \mathrm{CS}$ and $\mathrm{BnS}$ moieties during the deprotection of compounds $\mathbf{2 b}$ and $\mathbf{2} \mathbf{c}$ led us to modify the protecting group design to allow the deprotection of the phosphoryl and amino moieties under milder conditions. The diphenyl ester was replaced with the dimethyl or di-tert-butyl ester, and the 1-phenylthioureido moiety was changed to the $\mathrm{Cbz}$ or Boc moiety. The methodology for the preparation of precursors 7a-c, 8a-c and 9a-c is outlined in Scheme 2. 


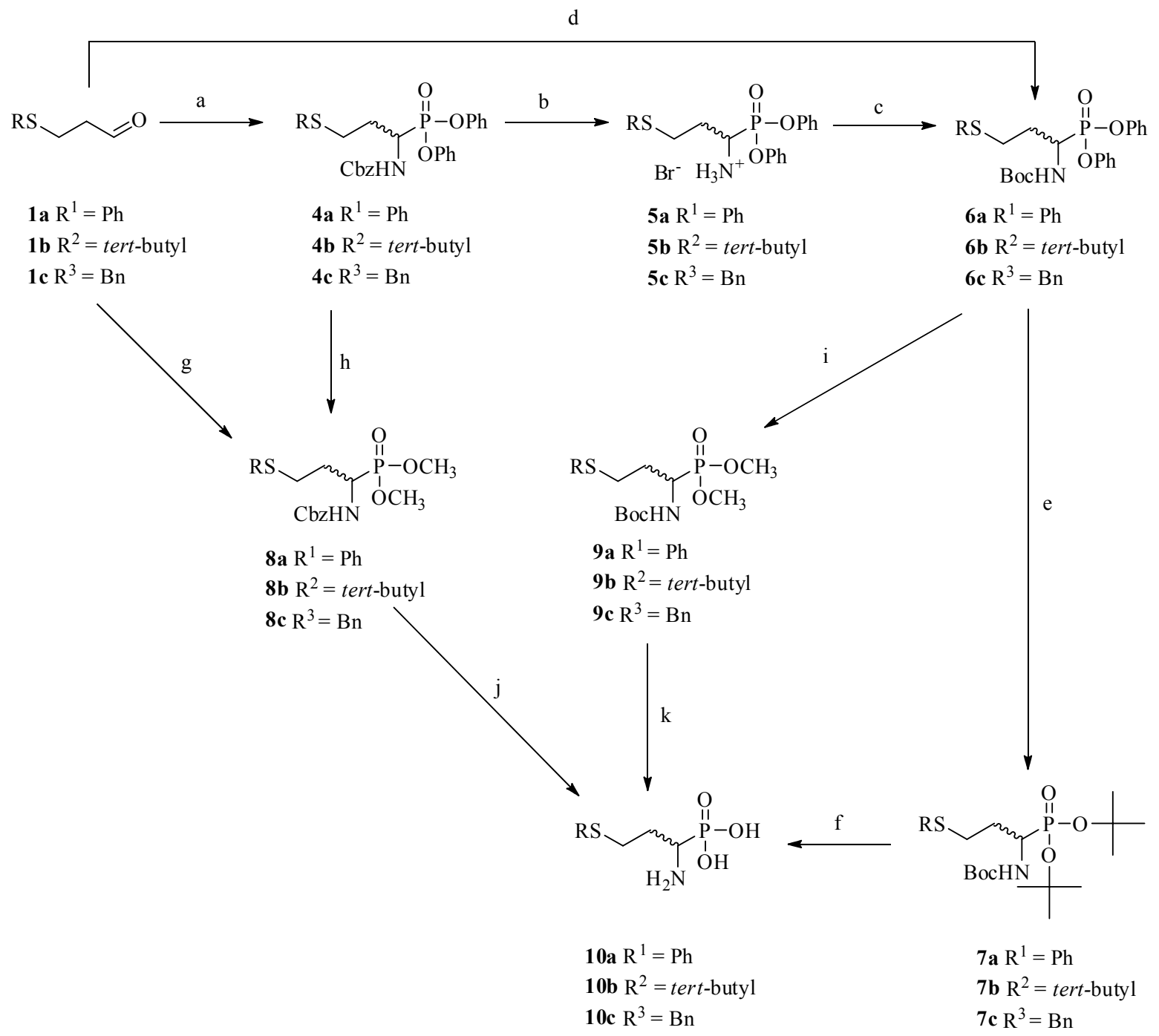

Scheme 2. Reactions, conditions and yields: (a) benzyl carbamate, triphenylphosphite, $\mathrm{AcOH}$, $80^{\circ} \mathrm{C}, 1 \mathrm{~h}, \mathrm{R}^{1}=\mathrm{Ph}(59 \%)$ for $\mathbf{4 a}, \mathrm{R}^{2}=$ tert-butyl $(57 \%)$ for $\mathbf{4 b}, \mathrm{R}^{3}=\mathrm{Bn}(55 \%)$ for $\mathbf{4 c}$; (b) $35 \%$ $\mathrm{HBr} / \mathrm{AcOH}, \mathrm{R}^{1}=\mathrm{Ph}(82 \%)$ for $\mathbf{5 a}, \mathrm{R}^{2}=$ tert-butyl $(78 \%)$ for $\mathbf{5 b}, \mathrm{R}^{3}=\mathrm{Bn}(75 \%)$ for $\mathbf{5} \mathbf{c}$; (c) $\mathrm{Boc}_{2} \mathrm{O}$, TEA, dichloromethane, $0^{\circ} \mathrm{C}$ for $1 \mathrm{~h}$, then rt overnight, $\mathrm{R}^{1}=\mathrm{Ph}(74 \%)$ for $\mathbf{6 a}, \mathrm{R}^{2}=$ tertbutyl $(65 \%)$ for $\mathbf{6 b}, \mathrm{R}^{3}=\mathrm{Bn}(75 \%)$ for $\mathbf{6 c}$; (d) tert-butyl carbamate, triphenyl phosphite, $\mathrm{TiCl}_{4}$, dichloromethane, rt, overnight, $\mathrm{R}_{1}=\mathrm{Ph}(52 \%)$ for $\mathbf{6} \mathbf{a}, \mathrm{R}^{2}=$ tert-butyl $(54 \%)$ for $\mathbf{6} \mathbf{b}, \mathrm{R}^{3}=\mathrm{Bn}$ (49\%) for $\mathbf{6 c}$; (e) potassium tert-butoxide, tert-butanol and dioxane, rt, overnight, $\mathrm{R}^{1}=\mathrm{Ph}(53 \%)$ for $7 \mathbf{a}, \mathrm{R}^{2}=$ tert-butyl $(59 \%)$ for $\mathbf{7 b}, \mathrm{R}^{3}=\mathrm{Bn}(60 \%)$ for $\mathbf{7} \mathbf{c}$; (f) TFA, dimethyl sulfide, DCM, water, rt, overnight, $\mathrm{R}^{1}=\mathrm{Ph}(73 \%)$ for $\mathbf{1 0 a}, \mathrm{R}^{2}=$ tert-butyl $(68 \%)$ for $\mathbf{1 0 b}, \mathrm{R}^{3}=\mathrm{Bn}(75 \%)$ for 10c; (g) benzyl carbamate, dimethyl phosphite, acetyl chloride $0^{\circ} \mathrm{C}$ for $1 \mathrm{~h}$, then $\mathrm{rt}$ overnight, $\mathrm{R}^{1}$ $=\mathrm{Ph}(31 \%)$ for $\mathbf{8} \mathbf{a}, \mathrm{R}^{2}=$ tert-butyl $(45 \%)$ for $\mathbf{8 b}, \mathrm{R}^{3}=\mathrm{Bn}(40 \%)$ for $\mathbf{8 c}$; (h) sodium methoxide, methanol and dioxane, rt overnight, $\mathrm{R}^{1}=\mathrm{Ph}(71 \%)$ for $\mathbf{8 a}, \mathrm{R}^{2}=$ tert-butyl $(66 \%)$ for $\mathbf{8 b}, \mathrm{R}^{3}=\mathrm{Bn}$ $(65 \%)$ for $8 c$; (i) sodium methoxide, methanol and dioxane, rt, overnight, $\mathrm{R}^{1}=\mathrm{Ph}(55 \%)$ for $9 \mathrm{a}$, $\mathrm{R}^{2}=$ tert-butyl $(64 \%)$ for $\mathbf{9 b}, \mathrm{R}^{3}=\mathrm{Bn}(57 \%)$ for $\mathbf{9 c}$; $(\mathrm{j}) 35 \% \mathrm{HBr} / \mathrm{AcOH}$ rt overnight then $1,2-$ 
epoxypropane, methanol, $\mathrm{R}^{1}=\mathrm{Ph}(69 \%)$ for $\mathbf{1 0 a}, \mathrm{R}^{2}=$ tert-butyl (trace) for $\mathbf{1 0 b}, \mathrm{R}^{3}=\mathrm{Bn}(62 \%)$ for 10c; (k) TMSBr, acetonitrile, rt for 48 hours, then TFA, dimethyl sulfide, DCM, water, rt, overnight, $\mathrm{R}^{1}=\mathrm{Ph}(50 \%)$ for $\mathbf{1 0 a}, \mathrm{R}^{2}=$ tert-butyl $(51 \%)$ for $\mathbf{1 0 b}, \mathrm{R}^{3}=\mathrm{Bn}(55 \%)$ for $\mathbf{1 0 c}$.

The Birum-Oleksyszyn condensation ${ }^{11,12}$ of aldehydes 1a-c with triphenyl phosphite and benzyl carbamate afforded Z-protected diphenyl phosphonates 4a-c, enabling access to the first precursors, dimethylesters 8a-c, via transesterification using sodium methanolate. Cleavage of the benzyloxycarbonyl group from compounds 4a-c was achieved by treatment with hydrobromic acid in glacial acetic acid to yield the corresponding salts 5a-c, which were subsequently converted to Boc-protected phosphonates 6a-c by reaction with triethylamine and Boc anhydride. The three-step synthetic sequence converting compounds 1a-c into 6a-c was successfully accomplished by the one-pot reaction of the aldehydes (1a-c) with triphenyl phosphite and tert-butyl carbamate in the presence of titanium tetrachloride as a Lewis acid. ${ }^{15}$ The subsequent transesterification of diphenyl esters 6a-c was accomplished by using either potassium tert-butanoate or sodium methanolate to afford precursors 7a-c and 9a-c, respectively. An alternative and straightforward route for the preparation of compounds 8a-8c was the reaction of benzyl carbamate and dimethyl phosphite with aldehydes 1a-1c, respectively, in acetyl chloride. $^{16}$

The final deprotection step, the treatment of precursors 8a and 8c with $35 \% \mathrm{HBr} / \mathrm{AcOH}$, furnished $10 \mathbf{a}$ and $10 \mathbf{c}$, respectively. In the case of compound $\mathbf{8 b}$, the $\mathrm{C}-\mathrm{S}$ bond underwent fission and only traces of $\mathbf{1 0 b}$ were isolated from the reaction mixture. We believe that the stability of the $\left(\mathrm{CH}_{3}\right)_{3} \mathrm{CS}$ moiety depends on the temperature during the workup. While debenzyloxycarbonylation of $\mathbf{4 b}$ in $35 \% \mathrm{HBr} / \mathrm{AcOH}$ proceeded smoothly at room temperature and gave a precipitate of $\mathbf{5 b}$ in $78 \%$ yield, the reaction from $\mathbf{8 b}$ to $\mathbf{1 0 b}$ required evaporation of the acids using a rotary evaporator at $60^{\circ} \mathrm{C}$ and caused the decomposition of the compound scaffold.

The most promising precursors 7a-c were treated with an acidic cleavage cocktail containing dimethyl sulfide (DMS) as a scavenger and afforded target compounds 10a-c in yields of approximately $70 \%$ after RP-HPLC purification. Alternatively, the two-step deprotection of 9a-c represents another possibility for the synthesis of $\mathbf{1 0 a}-\mathbf{c}$. The methyl esters were preferentially demethylated using TMSBr followed by acidic hydrolysis to removed the Boc group. Decreased yields (approximately 50\%) were achieved in this case.

\section{Conclusions}

From the literature ${ }^{7-9}$ and from our experimental results described above, we can deduce the following conclusions: (i) derivatives of phosphonohomocysteine with $S$-linked unbranched alkyl and phenyl substituents are inert towards hot and aqueous strong acids; (ii) BnS derivatives are stable in hydrobromic acid but only under non-aqueous conditions; (iii) the acidolability of 
the $\left(\mathrm{CH}_{3}\right)_{3} \mathrm{CS}$ moiety in trifluoroacetic acid is significantly reduced; and (iv) the observed order in the stability of the three substituents studied for use on the sulfur atom of phosphonohomocysteine in strong acid solutions is $\mathrm{Ph}>\mathrm{Bn}>$ tert-butyl. We hope that these proposed synthetic procedures describing the syntheses of phosphonohomocysteine precursors with di-tert-butylphosphoryl and tert-butyloxycarbonylamino groups might be helpful and might serve for the preparation of novel $S$-substituted phosphonohomocysteines.

\section{Experimental Section}

General Procedures. The reagents and solvents (Sigma-Aldrich-Fluka) used in this study were of analytical grade. The TLCs were performed on silica gel-coated aluminum plates (Fluka) using the following systems (v/v): chloroform-ethanol 99/1 (S1); chloroform-ethanol 98/2 (S2); chloroform-ethanol 95/5 (S3); chloroform-ethanol 90/10 (S4); isopropyl alcohol - concentrated aqueous ammonia - water 7/1/2 (S5); toluene-ethyl acetate 80/20 (S6). The compounds were visualized by exposure to UV light at $254 \mathrm{~nm}$, by ninhydrin application (amines show a dark blue color), by $1 \% \mathrm{KMnO}_{4}$ application (sulfides show a yellow color) and by application of a $1 \%(\mathrm{v} / \mathrm{v})$ ethanolic solution of 4-(4-nitrobenzyl)pyridine followed by heating and exposure to gaseous ammonia (the diesters of phosphonic acids show a blue color). Flash chromatography purifications were carried out on silica gel (40-63 $\mu \mathrm{m}$, Fluka). Preparative RP-HPLC chromatography was carried out using a C18 Luna column (Phenomenex, 250 x 21.2 mm, 10 $\mu \mathrm{m})$ at a flow rate $9 \mathrm{ml} / \mathrm{min}$. The solvents used were the following: solvent $\mathrm{A}-0.1 \% \mathrm{TFA}$ in water; and solvent $\mathrm{B}-80 \% \mathrm{CH}_{3} \mathrm{CN}, 0.1 \% \mathrm{TFA}$ in water. The following gradients were used: $\mathrm{G} 1$ : $\mathrm{t}=0 \min (90 \% \mathrm{~A}, 10 \% \mathrm{~B}), \mathrm{t}=30 \min (30 \% \mathrm{~A}, 70 \% \mathrm{~B}), \mathrm{t}=31 \min (90 \% \mathrm{~A}, 10 \% \mathrm{~B}) ; \mathrm{G} 2: \mathrm{t}=0$ $\min (59 \% \mathrm{~A}, 41 \% \mathrm{~B}), \mathrm{t}=10 \min (59 \% \mathrm{~A}, 41 \% \mathrm{~B}), \mathrm{t}=30 \min (54 \% \mathrm{~A}, 46 \% \mathrm{~B}), \mathrm{t}=31 \mathrm{~min}$ $(100 \% \mathrm{~B}), \mathrm{t}=34 \mathrm{~min}(59 \% \mathrm{~A}, 41 \% \mathrm{~B})$. Analytical RP-HPLC chromatography was carried out at a flow rate of $1 \mathrm{ml} / \mathrm{min}$ using a C18 Nucleosil column $(250 \times 4 \mathrm{~mm}, 5 \mu \mathrm{m})$ from Watrex (Praha, $\mathrm{CZ}$ ) using the same gradients and solvents. The eluted compounds were detected at $218 \mathrm{~nm}$. Melting points were determined on a Boetius block and are uncorrected. ${ }^{1} \mathrm{H}$ and ${ }^{13} \mathrm{C}$ NMR spectra were measured on a Bruker AVANCE-600 spectrometer $\left({ }^{1} \mathrm{H}\right.$ at $600.13 \mathrm{MHz},{ }^{13} \mathrm{C}$ at 150.9 $\mathrm{MHz}$ ) in $\mathrm{CDCl}_{3}, \mathrm{DMSO}-d_{6}, \mathrm{CD}_{3} \mathrm{OD}$ or $\mathrm{D}_{2} \mathrm{O}$ solution at $300 \mathrm{~K}$. The $2 \mathrm{D}-\mathrm{H}, \mathrm{H}-\mathrm{COSY}, 2 \mathrm{D}-\mathrm{H}, \mathrm{C}-$ HSQC and 2D-H,C-HMBC spectra were recorded and used for the structural assignments of proton and carbon signals. IR spectra were recorded on a Bruker IFS 55 Equinox apparatus. HRMS spectra were obtained on a FTMS mass spectrometer LTQ-orbitrap XL (Thermo Fisher, Bremen, Germany) in electrospray ionization mode or, in the case of HRMS (EI) spectra, on a GCT Premier (Waters). The purities of the target compounds were confirmed by elemental analysis $(\mathrm{C}, \mathrm{H}, \mathrm{N})$, and the experimental values differed from the calculated values by less than $0.4 \%$. 
3-(Phenylsulfanyl)propanal (1a). Thiophenol (27.5 g; $0.25 \mathrm{~mol})$ in $100 \mathrm{ml}$ dichlormethane was added dropwise over 30 minutes to an ice-cooled and stirred solution of acrolein (14 g; $0.25 \mathrm{~mol}$ ) and $10 \mathrm{ml}$ of triethylamine in $400 \mathrm{ml}$ dichlormethane. The ice bath was removed and stirring continued overnight at rt. All volatile materials were evaporated in vacuo and yellow residue was distilled under reduced pressure. Yield $32.7 \mathrm{~g}$ (79\%). Colorless liquid, bp $91-95{ }^{\circ} \mathrm{C} / 0.6-0.8$ torr (lit. ${ }^{17} 94-99{ }^{\circ} \mathrm{C} / 0.7$ torr). $\mathrm{R}_{\mathrm{f}}=0.64(\mathrm{~S} 2) .{ }^{1} \mathrm{H}$ NMR $\left(500 \mathrm{MHz} ; \mathrm{CDCl}_{3}\right): \delta_{\mathrm{H}} 2.77\left(2 \mathrm{H}, \mathrm{td},{ }^{3} J_{\mathrm{HH}}=7.1\right.$ and $\left.1.2 \mathrm{~Hz}, \mathrm{CH}_{2}-\mathrm{CO}\right), 3.19\left(2 \mathrm{H}, \mathrm{t},{ }^{3} \mathrm{~J}_{\mathrm{HH}}=7.1 \mathrm{~Hz}, \mathrm{CH}_{2}-\mathrm{S}\right), 7.22\left(1 \mathrm{H}_{\text {arom }}, \mathrm{m}, 1 \mathrm{CH}\right), 7.31\left(2 \mathrm{H}_{\text {arom, }}\right.$ $\mathrm{m}, 2 \mathrm{CH}), 7.36\left(2 \mathrm{H}_{\text {arom }}, \mathrm{m}, 2 \mathrm{CH}\right), 9.77\left(1 \mathrm{H}, \mathrm{t},{ }^{3} \mathrm{~J}_{\mathrm{HH}}=1.2 \mathrm{~Hz}, \mathrm{CH}=\mathrm{O}\right) .{ }^{13} \mathrm{C} \mathrm{NMR}(125.8 \mathrm{MHz}$; $\left.\mathrm{CDCl}_{3}\right): \delta_{\mathrm{C}} 26.39\left(1 \mathrm{C}, \mathrm{S}-\mathrm{CH}_{2}\right), 43.23\left(1 \mathrm{C}, \underline{\mathrm{CH}}_{2}-\mathrm{CO}\right), 126.66,129.09(2), 130.02(2)$ and 135.04 $\left(6 \mathrm{C}_{\text {arom }}, \mathrm{C}_{6} \mathrm{H}_{5}\right), 200.20(1 \mathrm{C}, \mathrm{CH}=\mathrm{O})$. IR (film, $\left.v_{\max }, \mathrm{cm}^{-1}\right) 1724$ vs $(\mathrm{C}=\mathrm{O}), 1584 \mathrm{~m}, 1482 \mathrm{~s}, 1440$ s, $692 \mathrm{~s}$ (ring). HRMS (EI) calc for $\mathrm{C}_{9} \mathrm{H}_{10} \mathrm{OS}[\mathrm{M}]^{+}$166.0452; found: 166.0451 .

3-(tert-Butylsulfanyl)propanal (1b). Aldehyde 1b was prepared in the same fashion as compound 1a by the reaction of acrolein (16.8 g; $0.3 \mathrm{~mol}), 2$-methyl-2-propanethiol (27.1 g; 0.3 mol) and $10 \mathrm{ml} \mathrm{TEA}$. Yield $32.7 \mathrm{~g}(75 \%)$. Colorless liquid, bp $65-67{ }^{\circ} \mathrm{C} / 7$ torr. $\mathrm{R}_{\mathrm{f}}=0.76(\mathrm{~S} 2)$. ${ }^{1} \mathrm{H}$ NMR $\left(600 \mathrm{MHz} ; \mathrm{CDCl}_{3}\right): \delta_{\mathrm{H}} 1.34\left(9 \mathrm{H}, \mathrm{s}, \mathrm{C}\left(\mathrm{CH}_{3}\right)_{3}\right), 2.72\left(2 \mathrm{H}, \mathrm{m}, \mathrm{CH}_{2}-\mathrm{CO}\right), 2.81(2 \mathrm{H}, \mathrm{m}$, $\left.\mathrm{CH}_{2}-\mathrm{S}\right), 9.78\left(1 \mathrm{H}, \mathrm{t},{ }^{3} J_{\mathrm{HH}}=1.3 \mathrm{~Hz}, \mathrm{CH}=\mathrm{O}\right) .{ }^{13} \mathrm{C} \mathrm{NMR}\left(150.9 \mathrm{MHz} ; \mathrm{CDCl}_{3}\right): \delta_{\mathrm{C}} 20.64(1 \mathrm{C}, \mathrm{S}-$ $\left.\mathrm{CH}_{2}\right), 30.75\left(3 \mathrm{C}, \mathrm{C}\left(\underline{\mathrm{CH}}_{3}\right)_{3}\right), 42.47\left(1 \mathrm{C},-\underline{\mathrm{C}}\left(\mathrm{CH}_{3}\right)_{3}\right), 43.61\left(1 \mathrm{C}, \underline{\mathrm{CH}}_{2}-\mathrm{CO}\right), 200.69$ (1C, $\left.\mathrm{CH}=\mathrm{O}\right)$. IR (film, $\left.v_{\max }, \mathrm{cm}^{-1}\right) 1726$ vs $(\mathrm{C}=\mathrm{O}), 1365 \mathrm{~s}\left(\mathrm{CH}_{3}\right), 1165 \mathrm{~s}\left(\mathrm{C}\left(\mathrm{CH}_{3}\right)_{3}\right)$. HRMS (EI) calc for $\mathrm{C}_{7} \mathrm{H}_{14} \mathrm{OS}$ $[\mathrm{M}]^{+}$146.0765; found: 146.0768 .

3-(Benzylsulfanyl)propanal (1c). Aldehyde 1c was prepared in the same fashion as compound $1 \mathrm{a}$ by the reaction of acrolein $(16.8 \mathrm{~g}$; $0.3 \mathrm{~mol})$, benzylmercaptan $(37.2 \mathrm{~g} ; 0.3 \mathrm{~mol})$ and $10 \mathrm{ml}$ of TEA. Yield $44 \mathrm{~g}(81 \%)$. Colorless liquid, bp $94-96{ }^{\circ} \mathrm{C} / 0.4$ torr (lit. ${ }^{18} 96-98{ }^{\circ} \mathrm{C} / 0.3$ torr). $\mathrm{R}_{\mathrm{f}}=0.76$ (S2). ${ }^{1} \mathrm{H}$ NMR $\left(600 \mathrm{MHz} ; \mathrm{CDCl}_{3}\right): \delta_{\mathrm{H}} 2.65\left(2 \mathrm{H}, \mathrm{m}, \mathrm{CH}_{2}-\mathrm{CO}\right), 2.69\left(2 \mathrm{H}, \mathrm{s}, \mathrm{CH}_{2}-\mathrm{S}\right), 3.73(2 \mathrm{H}, \mathrm{s}$, $\left.\mathrm{CH}_{2}-\mathrm{S}\right), 7.25\left(1 \mathrm{H}_{\text {arom }}, \mathrm{m}, 1 \mathrm{CH}\right), 7.31\left(4 \mathrm{H}_{\text {arom }}, \mathrm{m}, 4 \mathrm{CH}\right), 9.70\left(1 \mathrm{H}, \mathrm{t},{ }^{3} J_{\mathrm{HH}}=1.3 \mathrm{~Hz}, \mathrm{CH}=\mathrm{O}\right) .{ }^{13} \mathrm{C}$ NMR (150.9 MHz; $\left.\mathrm{CDCl}_{3}\right): \delta_{\mathrm{C}} 23.56\left(1 \mathrm{C}, \mathrm{S}-\mathrm{CH}_{2}\right), 36.45\left(1 \mathrm{C}, \mathrm{S}-\mathrm{CH}_{2}\right), 43.26\left(1 \mathrm{C}, \mathrm{CH}_{2}-\mathrm{CO}\right)$, 127.12, 128.55(2), 128.77(2) and $137.89\left(6 \mathrm{C}_{\text {arom }}, \mathrm{C}_{6} \mathrm{H}_{5}\right), 200.48(1 \mathrm{C}, \mathrm{CH}=\mathrm{O}) . \mathrm{IR}\left(\mathrm{CHCl}_{3}, v_{\max }\right.$, $\left.\mathrm{cm}^{-1}\right) 1724$ vs $(\mathrm{C}=\mathrm{O}), 1495 \mathrm{~m}, 1454 \mathrm{~m}, 703 \mathrm{~s}$ (ring). HRMS (EI) calc for $\mathrm{C}_{10} \mathrm{H}_{12} \mathrm{OS}[\mathrm{M}]^{+}$ 180.0609; found: 180.0617 .

Diphenyl [(R,S)-1-(3-phenylthioureido)-3-(phenylsulfanyl)propyl]phosphonate (2a). Compound 2a was prepared according to a modified protocol published by Kudzin and Stec ${ }^{7}$. A solution of triphenyl phosphite (13.7 g; $44 \mathrm{mmol})$, aldehyde 1a (8 g; $48.4 \mathrm{mmol})$ and $N$-phenyl thiourea $(6.7 \mathrm{~g} ; 44 \mathrm{mmol})$ in $50 \mathrm{ml}$ of glacial acetic acid was heated for 1 hour at $80^{\circ} \mathrm{C}$. The acetic acid was evaporated in vacuo, and the residue was dissolved in $100 \mathrm{ml}$ of methanol. The solution was left to stand overnight at $-20^{\circ} \mathrm{C}$ and afforded white crystals, which were removed by filtration and washed with petroleum ether. The pure product was obtained by recrystallization from a mixture of chloroform-methanol. Yield $14 \mathrm{~g}(59 \%)$. White solid, mp $116-119{ }^{\circ} \mathrm{C} . \mathrm{R}_{\mathrm{f}}=$ 0.73 (S2). ${ }^{1} \mathrm{H}$ NMR (600 MHz; d $\left.{ }_{6} \mathrm{DMSO}\right): \delta_{\mathrm{H}} 2.14(1 \mathrm{H}, \mathrm{m}, \mathrm{C}-\mathrm{CHaHb}-\mathrm{C}), 2.23(1 \mathrm{H}, \mathrm{m}, \mathrm{C}-$ $\mathrm{CHaHb}-\mathrm{C}), 3.06\left(1 \mathrm{H}, \mathrm{ddd},{ }^{2} J_{\mathrm{HH}}=13.6,{ }^{3} J_{\mathrm{HH}}=8.2\right.$ and $\left.7.3 \mathrm{~Hz}, \mathrm{~S}-\underline{\mathrm{HaHb}}\right), 3.18\left(1 \mathrm{H}, \mathrm{ddd},{ }^{2} J_{\mathrm{HH}}=\right.$ $13.6,{ }^{3} J_{\mathrm{HH}}=8.5$ and $\left.4.8 \mathrm{~Hz}, \mathrm{~S}-\mathrm{CHaHb}\right), 5.85\left(1 \mathrm{H}, \mathrm{dtd},{ }^{3} J_{\mathrm{HP}}=17.0,{ }^{3} J_{\mathrm{HH}}=10.2,9.8\right.$ and $3.6 \mathrm{~Hz}$, $\mathrm{N}-\mathrm{CH}-\mathrm{P}), 7.14\left(1 \mathrm{H}_{\text {arom }}, \mathrm{m}, 1 \mathrm{CH}\right), 7.17\left(4 \mathrm{H}_{\text {arom }}, \mathrm{m}, 4 \mathrm{CH}\right), 7.21\left(1 \mathrm{H}_{\text {arom }}, \mathrm{m}, 1 \mathrm{CH}\right), 7.23\left(2 \mathrm{H}_{\text {arom }}, \mathrm{m}\right.$, 
2CH), $7.33\left(2 \mathrm{H}_{\text {arom }}, \mathrm{m}, 2 \mathrm{CH}\right), 7.34\left(2 \mathrm{H}_{\text {arom }}, \mathrm{m}, 2 \mathrm{CH}\right), 7.36\left(2 \mathrm{H}_{\text {arom }}, \mathrm{m}, 2 \mathrm{CH}\right), 7.39\left(4 \mathrm{H}_{\text {arom }}, \mathrm{m}\right.$, $4 \mathrm{CH}), 7.48\left(2 \mathrm{H}_{\text {arom }}, \mathrm{m}, 2 \mathrm{CH}\right), 8.36\left(1 \mathrm{H}, \mathrm{d},{ }^{3} \mathrm{~J}_{\mathrm{HH}}=9.8, \mathrm{NH}\right), 9.80(1 \mathrm{H}, \mathrm{s}, \mathrm{NH}) .{ }^{13} \mathrm{C}$ NMR (150.9 MHz; d $\left.{ }_{6} \mathrm{DMSO}\right): \delta_{\mathrm{C}} 28.83\left(1 \mathrm{C}, \mathrm{d},{ }^{3} J_{\mathrm{CP}}=15.6 \mathrm{~Hz}, \mathrm{~S}-\mathrm{CH}_{2}\right), 30.01\left(1 \mathrm{C}, \mathrm{d},{ }^{2} J_{\mathrm{CP}}=4.3 \mathrm{~Hz}, \mathrm{C}-\mathrm{CH}_{2}-\right.$ C), $50.02\left(1 \mathrm{C}, \mathrm{d},{ }^{1} J_{\mathrm{CP}}=156.6 \mathrm{~Hz}, \mathrm{~N}-\mathrm{CH}-\mathrm{P}\right), 120.68\left(2 \mathrm{C}, \mathrm{d},{ }^{3} J_{\mathrm{CP}}=3.8 \mathrm{~Hz}\right.$, ortho- $\left.\mathrm{CH}_{\text {arom }}\right), 120.78$ $\left(2 \mathrm{C}, \mathrm{d},{ }^{3} J_{\mathrm{CP}}=3.8 \mathrm{~Hz}\right.$, ortho- $\left.\mathrm{CH}_{\text {arom }}\right), 123.32(2), 124.75,125.67,125.72,126.14,128.61(2)$, 128.81(2), 129.39(2), 130.14(2) and 130.20(2) (16C, $\left.\mathrm{CH}_{\text {arom }}\right), 135.68$ (1C, ipso- $\left.\mathrm{C}_{\text {arom }}\right), 139.36$ (1C, ipso- $\left.\mathrm{C}_{\mathrm{arom}}\right), 149.82\left(\mathrm{~d},{ }^{2} J_{\mathrm{CP}}=9.5 \mathrm{~Hz}\right.$, ipso- $\left.\mathrm{C}_{\mathrm{arom}}\right), 150.07\left(\mathrm{~d},{ }^{2} J_{\mathrm{CP}}=9.6 \mathrm{~Hz}\right.$, ipso- $\left.\mathrm{C}_{\mathrm{arom}}\right)$, 181.89 (1C, N-CS-N). IR (KBr, $\left.v_{\max }, \mathrm{cm}^{-1}\right) 3330 \mathrm{~m}(\mathrm{NH}) ; 1538 \mathrm{vs}, 1355 \mathrm{~m}, 1326 \mathrm{~s}(\mathrm{HNC}=\mathrm{S})$; 1230 vs $(\mathrm{P}=\mathrm{O}) ; 1210$ vs, 1188 vs, $1163 \mathrm{~s}(\mathrm{Ph}-\mathrm{OP}) ; 954$ vs $(\mathrm{P}-\mathrm{OPh}) ; 1589 \mathrm{~m}, 1489$ vs, $1025 \mathrm{~m}$, $1008 \mathrm{~m}, 760 \mathrm{~s}, 690 \mathrm{~s}$ (ring). HRMS (ESI) calc for $\mathrm{C}_{28} \mathrm{H}_{28} \mathrm{O}_{3} \mathrm{~N}_{2} \mathrm{PS}_{2}[\mathrm{M}+1]^{+}$535.12735; found: 535.12768 .

Diphenyl [(R,S)-1-(3-phenylthioureido)-3-(tert-butylsulfanyl)propyl]phosphonate (2b). Phosphonate $\mathbf{2 b}$ was prepared in the same manner as compound $\mathbf{2 a}$ by the reaction of triphenyl phosphite (9.3 g; $30 \mathrm{mmol})$, aldehyde $1 \mathbf{b}(4.8 \mathrm{~g} ; 33 \mathrm{mmol})$ and $N$-phenyl thiourea (4.6 g; 30 mmol). Yield $7 \mathrm{~g}(45 \%)$. White solid, $\mathrm{mp} 134-135^{\circ} \mathrm{C}$ (lit. $\left.{ }^{7} 134-135^{\circ} \mathrm{C}\right) . \mathrm{R}_{\mathrm{f}}=0.73(\mathrm{~S} 2) .{ }^{1} \mathrm{H}$ NMR (600 MHz; d $\left.{ }_{6} \mathrm{DMSO}\right): \delta_{\mathrm{H}} 1.26\left(9 \mathrm{H}, \mathrm{s}, \mathrm{C}\left(\mathrm{CH}_{3}\right)_{3}\right), 2.09$ (1H, m, C-CHaHb-C), 2.19 (1H, m, CCHaHb-C), $2.66\left(1 \mathrm{H}, \mathrm{ddd},{ }^{2} J_{\mathrm{HH}}=12.8,{ }^{3} J_{\mathrm{HH}}=9.1\right.$ and $\left.6.9 \mathrm{~Hz}, \mathrm{~S}-\mathrm{CHaHb}\right), 2.71\left(1 \mathrm{H}, \mathrm{ddd},{ }^{2} J_{\mathrm{HH}}=\right.$ $12.8,{ }^{3} J_{\mathrm{HH}}=9.2$ and $\left.5.3 \mathrm{~Hz}, \mathrm{~S}-\mathrm{CHaHb}\right), 5.76\left(1 \mathrm{H}, \mathrm{dtd},{ }^{3} J_{\mathrm{HP}}=17.0,{ }^{3} J_{\mathrm{HH}}=9.7,9.7\right.$ and $4.0 \mathrm{~Hz}, \mathrm{~N}-$ CH-P), $7.14\left(1 \mathrm{H}_{\text {arom }}, \mathrm{m}, 1 \mathrm{CH}\right), 7.20\left(2 \mathrm{H}_{\text {arom }}, \mathrm{m}, 2 \mathrm{CH}\right), 7.24\left(4 \mathrm{H}_{\text {arom }}, \mathrm{m}, 4 \mathrm{CH}\right), 7.33\left(2 \mathrm{H}_{\text {arom }}, \mathrm{m}\right.$, $2 \mathrm{CH}), 7.41\left(4 \mathrm{H}_{\text {arom }}, \mathrm{m}, 4 \mathrm{CH}\right), 7.48\left(2 \mathrm{H}_{\text {arom }}, \mathrm{m}, 2 \mathrm{CH}\right), 8.33\left(1 \mathrm{H}, \mathrm{d},{ }^{3} J_{\mathrm{HH}}=9.7, \mathrm{NH}\right), 9.77(1 \mathrm{H}, \mathrm{s}$, $\mathrm{NH}) .{ }^{13} \mathrm{C}$ NMR (150.9 MHz; d $\left.{ }_{6} \mathrm{DMSO}\right): \delta_{\mathrm{C}} 24.35\left(1 \mathrm{C}, \mathrm{d},{ }^{3} J_{\mathrm{CP}}=14.5 \mathrm{~Hz}, \mathrm{~S}-\mathrm{CH}_{2}\right), 30.98(3 \mathrm{C}$, $\left.\mathrm{C}\left(\underline{\mathrm{CH}}_{3}\right)_{3}\right), 31.42\left(1 \mathrm{C}, \mathrm{d},{ }^{2} J_{\mathrm{CP}}=4.0 \mathrm{~Hz}, \mathrm{C}-\underline{\mathrm{CH}}_{2}-\mathrm{C}\right), 42.38\left(1 \mathrm{C}, \underline{\mathrm{C}}\left(\mathrm{CH}_{3}\right)_{3}\right), 50.44\left(1 \mathrm{C}, \mathrm{d},{ }^{1} J_{\mathrm{CP}}=\right.$ $156.0 \mathrm{~Hz}, \mathrm{~N}-\mathrm{CH}-\mathrm{P}), 120.68\left(2 \mathrm{C}, \mathrm{d},{ }^{3} J_{\mathrm{CP}}=3.7 \mathrm{~Hz}\right.$, ortho- $\left.\mathrm{CH}_{\text {arom }}\right), 120.82\left(2 \mathrm{C}, \mathrm{d},{ }^{3} J_{\mathrm{CP}}=3.8 \mathrm{~Hz}\right.$, ortho- $\mathrm{CH}_{\text {arom }}$ ), 123.25(2), 124.69, 125.63, 125.72, 128.78(2), 130.12(2) and 130.21(2) (11C, $\mathrm{CH}_{\text {arom}}$ ), 139.41 (1C, ipso- $\left.\mathrm{C}_{\text {arom }}\right), 149.90\left(1 \mathrm{C}, \mathrm{d},{ }^{2} J_{\mathrm{CP}}=9.5 \mathrm{~Hz}\right.$, ipso- $\left.\mathrm{C}_{\text {arom }}\right), 150.13\left(1 \mathrm{C}, \mathrm{d},{ }^{2} J_{\mathrm{CP}}=\right.$ $9.6 \mathrm{~Hz}$, ipso- $\left.\mathrm{C}_{\text {arom}}\right), 181.81(1 \mathrm{C}, \mathrm{N}-\mathrm{CS}-\mathrm{N}) . \mathrm{IR}\left(\mathrm{KBr}, v_{\max }, \mathrm{cm}^{-1}\right) 3317 \mathrm{~s}(\mathrm{NH}) ; 1537 \mathrm{vs}, 1348 \mathrm{~s}$, $1321 \mathrm{~s}(\mathrm{HNC}=\mathrm{S}) ; 1227$ vs $(\mathrm{P}=\mathrm{O}) ; 1211$ vs, 1186 vs, $1163 \mathrm{~s}(\mathrm{Ph}-\mathrm{OP}) ; 958 \mathrm{vs}(\mathrm{P}-\mathrm{OPh}) ; 1591 \mathrm{~s}$, 1489 vs, 1453 m, 1026 m, 1008 m, 763 s, 689 s (ring); $2961 \mathrm{~m}\left(\mathrm{CH}_{3}\right)$. HRMS (ESI) calc for $\mathrm{C}_{26} \mathrm{H}_{32} \mathrm{O}_{3} \mathrm{~N}_{2} \mathrm{PS}_{2}[\mathrm{M}+1]^{+}$515.15865; found: 515.15853 .

\section{Diphenyl [(R,S)-1-(3-phenylthioureido)-3-(benzylsulfanyl)propyl]phosphonate}

(2c). Phosphonate 2c was prepared in the same manner as compound 2a by the reaction of triphenyl phosphite $(9.3 \mathrm{~g} ; 30 \mathrm{mmol})$, aldehyde 1c $(5.4 \mathrm{~g} ; 33 \mathrm{mmol})$ and $N$-phenyl thiourea (4.6 g; 30 mmol). Yield $9 \mathrm{~g}(55 \%)$. White solid, mp 98-99 ${ }^{\circ} \mathrm{C}$ (lit. $\left.{ }^{7} 99-100{ }^{\circ} \mathrm{C}\right) . \mathrm{R}_{\mathrm{f}}=0.37(\mathrm{~S} 1) .{ }^{1} \mathrm{H} \mathrm{NMR}$ $\left(600 \mathrm{MHz} ; \mathrm{CDCl}_{3}\right): \delta_{\mathrm{H}} 1.84(1 \mathrm{H}, \mathrm{m}, \mathrm{C}-\mathrm{C} \underline{\mathrm{HaHb}}-\mathrm{C}), 2.20(1 \mathrm{H}, \mathrm{m}, \mathrm{C}-\mathrm{CHaHb}-\mathrm{C}), 2.58\left(2 \mathrm{H}, \mathrm{bt},{ }^{3} J_{\mathrm{HH}}\right.$ $\left.=7.5 \mathrm{~Hz}, \mathrm{~S}-\mathrm{CH}_{2}\right), 3.67\left(1 \mathrm{H}, \mathrm{d},{ }^{2} J_{\mathrm{HH}}=13.5, \mathrm{~S}-\mathrm{CHaHb}\right), 3.70\left(1 \mathrm{H}, \mathrm{d},{ }^{2} J_{\mathrm{HH}}=13.5, \mathrm{~S}-\mathrm{CHaHb}\right), 5.86$ $(1 \mathrm{H}, \mathrm{m}, \mathrm{N}-\mathrm{CH}-\mathrm{P}), 7.03\left(2 \mathrm{H}_{\text {arom }}, \mathrm{m}, 2 \mathrm{CH}\right), 7.06(1 \mathrm{H}, \mathrm{b}, \mathrm{NH}), 7.10\left(1 \mathrm{H}_{\text {arom }}, \mathrm{m}, 1 \mathrm{CH}\right), 7.12\left(2 \mathrm{H}_{\text {arom, }}\right.$ $\mathrm{m}, 2 \mathrm{CH}), 7.14\left(2 \mathrm{H}_{\text {arom }}, \mathrm{m}, 2 \mathrm{CH}\right), 7.16\left(1 \mathrm{H}_{\text {arom }}, \mathrm{m}, 1 \mathrm{CH}\right), 7.19\left(1 \mathrm{H}_{\text {arom }}, \mathrm{m}, 1 \mathrm{CH}\right), 7.20\left(2 \mathrm{H}_{\text {arom }}, \mathrm{m}\right.$, $2 \mathrm{CH}), 7.22\left(3 \mathrm{H}_{\text {arom }}, \mathrm{m}, 1 \mathrm{CH}\right), 7.26\left(4 \mathrm{H}_{\text {arom }}, \mathrm{m}, 4 \mathrm{CH}\right), 7.31\left(2 \mathrm{H}_{\text {arom }}, \mathrm{m}, 2 \mathrm{CH}\right), 8.52(1 \mathrm{H}, \mathrm{b}, \mathrm{NH})$. ${ }^{13} \mathrm{C}$ NMR (150.9 MHz; $\left.\mathrm{CDCl}_{3}\right): \delta_{\mathrm{C}} 27.34\left(1 \mathrm{C}, \mathrm{d},{ }^{3} J_{\mathrm{CP}}=14.5 \mathrm{~Hz}, \mathrm{~S}-\mathrm{CH}_{2}\right), 30.47\left(1 \mathrm{C}, \mathrm{C}-\mathrm{CH}_{2}-\mathrm{C}\right)$, $36.26\left(1 \mathrm{C}, \mathrm{S}-\mathrm{CH}_{2}\right), 50.42\left(1 \mathrm{C}, \mathrm{d},{ }^{1} J_{\mathrm{CP}}=156.1 \mathrm{~Hz}, \mathrm{~N}-\mathrm{CH}-\mathrm{P}\right), 120.41\left(2 \mathrm{C}, \mathrm{d},{ }^{3} J_{\mathrm{CP}}=3.9 \mathrm{~Hz}\right.$, ortho- 
$\left.\mathrm{CH}_{\text {arom }}\right), 120.57\left(2 \mathrm{C}, \mathrm{d},{ }^{3} J_{\mathrm{CP}}=4.1 \mathrm{~Hz}\right.$, ortho $\left.-\mathrm{CH}_{\text {arom }}\right), 124.49(2), 124.64,125.83,126.35,126.97$, 128.47(3), 128.84(2), 129.27(2), 129.74 and 129.98(2) (16C, $\left.\mathrm{CH}_{\text {arom }}\right), 138.02$ (1C, ipso- $\left.\mathrm{C}_{\text {arom}}\right)$, $149.40\left(1 \mathrm{C}, \mathrm{d},{ }^{2} J_{\mathrm{CP}}=10.5 \mathrm{~Hz}\right.$, ipso-C $\left.\mathrm{C}_{\mathrm{arom}}\right), 150.08\left(1 \mathrm{C}, \mathrm{d},{ }^{2} J_{\mathrm{CP}}=10.1 \mathrm{~Hz}\right.$, ipso-C $\left.\mathrm{C}_{\text {arom }}\right), 181.88$ $(1 \mathrm{C}, \mathrm{N}-\mathrm{CS}-\mathrm{N})$. IR $\left(\mathrm{KBr}, v_{\max }, \mathrm{cm}^{-1}\right) 3310 \mathrm{~s}(\mathrm{NH}) ; 1537 \mathrm{vs}, 1347 \mathrm{~s}, 1320 \mathrm{~s}(\mathrm{HNC}=\mathrm{S}) ; 1226 \mathrm{vs}$ $(\mathrm{P}=\mathrm{O}) ; 1207 \mathrm{vs}, 1186 \mathrm{vs}, 1162 \mathrm{~s}(\mathrm{Ph}-\mathrm{OP}) ; 958 \mathrm{vs}(\mathrm{P}-\mathrm{OPh}) ; 1590 \mathrm{~s}, 1489 \mathrm{vs}, 1452 \mathrm{~m}, 1025 \mathrm{~m}$, 1009 m, 768 s, 689 s (ring). HRMS (ESI) calc for $\mathrm{C}_{29} \mathrm{H}_{30} \mathrm{O}_{3} \mathrm{~N}_{2} \mathrm{PS}_{2}[\mathrm{M}+1]^{+}$549.1430; found: 549.1426.

1-(R,S), 8-(R,S)-Diamino-4,5-dithiaoctane-1,8-diphosphonic acid (3). Phosphonohomocystine was prepared by acid hydrolysis of $\mathbf{2 b}(6 \mathrm{~g} ; 11.7 \mathrm{mmol})$ as described earlier by Kudzin and $\mathrm{Stec}^{7}$. Yield $1.3 \mathrm{~g}(65 \%)$. White solid, mp $267-269{ }^{\circ} \mathrm{C}$ (lit. $\left.{ }^{7} 271-273{ }^{\circ} \mathrm{C}\right) . \mathrm{R}_{\mathrm{f}}=0.05$ (S4). Calcd. for $\mathrm{C}_{6} \mathrm{H}_{18} \mathrm{~N}_{2} \mathrm{O}_{6} \mathrm{P}_{2} \mathrm{~S}_{2}$ (340.3) C $21.18 \%$, H 5.34\%, N 8.23\%. Found: C 21. 27\%, H 5.33\%, N 8.01\%. ${ }^{1} \mathrm{H}$ NMR (600 MHz; $\left.\mathrm{D}_{2} \mathrm{O}+\mathrm{NaOD}\right)$ : (signals of 2 diatereoisomers overlapped) $\delta_{\mathrm{H}} 2.07(1 \mathrm{H}, \mathrm{m}$, C-C $\underline{H a H b}-\mathrm{C}), 2.32$ (1H, m, C-CHab-C), 2.87 (1H, m, S-C $\underline{\mathrm{HaHb}}), 2.99$ (1H, m, S-CHaHb), $3.16(1 \mathrm{H}, \mathrm{m}, \mathrm{N}-\mathrm{CH}-\mathrm{P}), 7.03(2 \mathrm{H}, \mathrm{m}, 2 \mathrm{CH}) .{ }^{13} \mathrm{C} \mathrm{NMR}\left(150.9 \mathrm{MHz} ; \mathrm{D}_{2} \mathrm{O}+\mathrm{NaOD}\right)$ : (signals of 2 diatereoisomers $\sim 1: 1) \delta_{\mathrm{C}} 31.63$ and $31.65\left(1 \mathrm{C}, \mathrm{C}-\underline{C H}_{2}-\mathrm{C}\right), 37.41$ and $37.55\left(1 \mathrm{C}, \mathrm{d},{ }^{3} J_{\mathrm{CP}}=10.0\right.$ $\left.\mathrm{Hz}, \mathrm{S}-\mathrm{CH}_{2}\right), 51.86$ and $51.93\left(1 \mathrm{C}, \mathrm{d},{ }^{1} J_{\mathrm{CP}}=132.4 \mathrm{~Hz}, \mathrm{~N}-\mathrm{CH}-\mathrm{P}\right)$. IR (KBr, $\left.v_{\max }, \mathrm{cm}^{-1}\right) 2920 \mathrm{vbr}$, $1628 \mathrm{~s}, 1535 \mathrm{~s}\left(\mathrm{NH}_{3}^{+}\right)$; $1171 \mathrm{vs,} 1035$ vs $\left(\mathrm{PO}_{2}^{-}\right), 928 \mathrm{~s}(\mathrm{POH})$. HRMS (ESI) calc for $\mathrm{C}_{6} \mathrm{H}_{17} \mathrm{O}_{6} \mathrm{~N}_{2} \mathrm{P}_{2} \mathrm{~S}_{2}[\mathrm{M}-1]^{+} 339.00087$; found: 339.00081 .

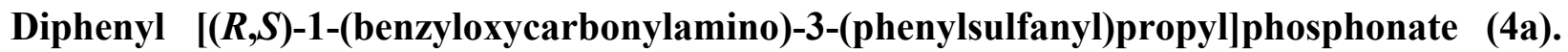
Compound $4 \mathbf{a}$ was prepared according to the procedure described by Vo-Quang et al. ${ }^{19}$ Benzyl carbamate (20.6 g; $0.14 \mathrm{~mol})$, triphenylphosphite $(43.4 \mathrm{~g} ; 0.14 \mathrm{~mol})$ and 3(phenylsulfanyl)propanal 1a $(24.4 \mathrm{~g} ; 0.15 \mathrm{~mol})$ were stirred for 1.5 hours at $80^{\circ} \mathrm{C}$ in glacial acetic acid $(100 \mathrm{ml})$. The acetic acid was evaporated in vacuo, and the residue was dissolved in $200 \mathrm{ml}$ of methanol. The solution was left to stand overnight at $-20^{\circ} \mathrm{C}$ to afford white crystals, which were removed by filtration and washed with petroleum ether. The pure product was obtained by recrystallisation from a mixture of chloroform-methanol. Yield $43 \mathrm{~g}$ (59\%). White solid, mp 106-107 ${ }^{\circ} \mathrm{C}$ (lit. $\left.{ }^{19} 106-107{ }^{\circ} \mathrm{C}\right) . \mathrm{R}_{\mathrm{f}}=0.27$ (S1). ${ }^{1} \mathrm{H}$ NMR $\left(500 \mathrm{MHz} ; \mathrm{CDCl}_{3}\right): \delta_{\mathrm{H}} 2.05$ $(1 \mathrm{H}, \mathrm{m}, \mathrm{C}-\mathrm{CHaHb}-\mathrm{C}), 2.31(1 \mathrm{H}, \mathrm{m}, \mathrm{C}-\mathrm{CHaHb}-\mathrm{C}), 2.99\left(1 \mathrm{H}, \mathrm{ddd},{ }^{2} J_{\mathrm{HH}}=13.2,{ }^{3} J_{\mathrm{HH}}=8.7\right.$ and 7.2 $\mathrm{Hz}, \mathrm{S}-\mathrm{CHaHb}), 3.11\left(1 \mathrm{H}, \mathrm{ddd},{ }^{2} J_{\mathrm{HH}}=13.2,{ }^{3} J_{\mathrm{HH}}=8.9\right.$ and $\left.4.8 \mathrm{~Hz}, \mathrm{~S}-\mathrm{CHaHb}\right), 4.68\left(1 \mathrm{H}, \mathrm{dtd},{ }^{3} J_{\mathrm{HP}}\right.$ $=17.9,{ }^{3} J_{\mathrm{HH}}=10.6,10.2$ and $\left.3.8 \mathrm{~Hz}, \mathrm{~N}-\mathrm{CH}-\mathrm{P}\right), 5.11\left(1 \mathrm{H}, \mathrm{d},{ }^{2} J_{\mathrm{HH}}=12.2 \mathrm{~Hz}, \mathrm{CHaHb}-\mathrm{O}\right), 5.14$ $\left(1 \mathrm{H}, \mathrm{d},{ }^{2} J_{\mathrm{HH}}=12.2 \mathrm{~Hz}, \mathrm{CHaHb}-\mathrm{O}\right), 5.34\left(1 \mathrm{H}, \mathrm{bd},{ }^{3} J_{\mathrm{HH}}=10.2 \mathrm{~Hz}, \mathrm{NH}\right), 7.06-7.34\left(20 \mathrm{H}_{\mathrm{arom}}, \mathrm{m}\right.$, 20CH). ${ }^{13} \mathrm{C}$ NMR (125.8 MHz; $\left.\mathrm{CDCl}_{3}\right): \delta_{\mathrm{C}} 30.04\left(1 \mathrm{C}, \mathrm{d},{ }^{2} J_{\mathrm{CP}}=6.0 \mathrm{~Hz}, \mathrm{C}-\underline{C H}_{2}-\mathrm{C}\right), 30.24(1 \mathrm{C}, \mathrm{d}$, $\left.{ }^{3} J_{\mathrm{CP}}=18.9 \mathrm{~Hz}, \mathrm{~S}-\mathrm{CH}_{2}\right), 47.62\left(1 \mathrm{C}, \mathrm{d},{ }^{1} J_{\mathrm{CP}}=198.4 \mathrm{~Hz}, \mathrm{~N}-\mathrm{CH}-\mathrm{P}\right), 67.45\left(1 \mathrm{C}, \mathrm{CH}_{2}-\mathrm{O}\right), 120.33$ $\left(2 \mathrm{C}, \mathrm{d},{ }^{3} J_{\mathrm{CP}}=5.4 \mathrm{~Hz}\right.$, ortho- $\left.\mathrm{CH}_{\text {arom }}\right), 120.47\left(2 \mathrm{C}, \mathrm{d},{ }^{3} J_{\mathrm{CP}}=5.4 \mathrm{~Hz}\right.$, ortho- $\left.\mathrm{CH}_{\text {arom }}\right), 125.33$, $125.45,126.47,128.17(2), 128.30(2), 128.55(2), 129.02(2), 129.71,129.82(2)$ and 130.01(2) (16C, $\left.\mathrm{CH}_{\text {arom }}\right), 135.98$ and $135.23\left(2 \mathrm{C}, \mathrm{C}_{\text {arom }}\right), 149.89\left(1 \mathrm{C}, \mathrm{d},{ }^{2} J_{\mathrm{CP}}=12.1 \mathrm{~Hz}\right.$, ipso- $\left.\mathrm{C}_{\text {arom }}\right), 150.13$ $\left(1 \mathrm{C}, \mathrm{d},{ }^{3} J_{\mathrm{CP}}=12.2 \mathrm{~Hz}\right.$, ipso- $\left.\mathrm{C}_{\text {arom }}\right), 155.86\left(1 \mathrm{C}, \mathrm{d},{ }^{3} J_{\mathrm{CP}}=8.2 \mathrm{~Hz}, \mathrm{~N}-\mathrm{CO}-\mathrm{O}\right) . \mathrm{IR}\left(\mathrm{KBr}, v_{\max }, \mathrm{cm}^{-1}\right)$ 3261 s $(\mathrm{NH}) ; 1545$ s $(\mathrm{CONH}) ; 1715$ vs $(\mathrm{C}=\mathrm{O}) ; 1251 \mathrm{~s}(\mathrm{P}=\mathrm{O}) ; 1196 \mathrm{~s}(\mathrm{Ph}-\mathrm{OP}) ; 955$ vs $(\mathrm{P}-\mathrm{OPh})$; $1587 \mathrm{~m}, 1491 \mathrm{~s}, 1457 \mathrm{~m}, 1436 \mathrm{~m}, 1070 \mathrm{~m}, 1481 \mathrm{~s}, 765 \mathrm{~m}, 688 \mathrm{~s}$ (ring). HRMS (EI) calc for $\mathrm{C}_{29} \mathrm{H}_{29} \mathrm{O}_{5} \mathrm{NPS}[\mathrm{M}+1]^{+}$534.1499; found: 534.1495. 
Diphenyl [(R,S)-1-(benzyloxycarbonylamino)-3-(tert-butylsulfanyl)propyl]phosphonate (4b). Using the procedure as outlined for $\mathbf{4 a}$, compound $\mathbf{4 b}$ was prepared from benzyl carbamate (18.9 g; $0.125 \mathrm{~mol})$, triphenylphosphite $(38.8 \mathrm{~g} ; 0.125 \mathrm{~mol}$ ) and 3-(tert-butylsulfanyl)propanal (1b) $(20 \mathrm{~g} ; 0.137 \mathrm{~mol})$. The residue was triturated at $-20{ }^{\circ} \mathrm{C}$ using a mixture of diethyl etherpetroleum ether $(200 \mathrm{ml} ; 1: 4)$ to afford a brown material, which was isolated by filtration and purified by crystallization from a mixture of chloroform-petroleum ether. Yield $36.5 \mathrm{~g}$ (57\%). White solid, mp 81-83 ${ }^{\circ} \mathrm{C} . \mathrm{R}_{\mathrm{f}}=0.46(\mathrm{~S} 2) .{ }^{1} \mathrm{H} \mathrm{NMR}\left(600 \mathrm{MHz} ; \mathrm{CDCl}_{3}\right): \delta_{\mathrm{H}} 1.29(9 \mathrm{H}, \mathrm{s}$, $\left.\mathrm{C}\left(\mathrm{CH}_{3}\right)_{3}\right), 2.03(1 \mathrm{H}, \mathrm{m}, \mathrm{C}-\mathrm{CHaHb}-\mathrm{C}), 2.28(1 \mathrm{H}, \mathrm{m}, \mathrm{C}-\mathrm{CHaHb}-\mathrm{C}), 2.63\left(1 \mathrm{H}, \mathrm{ddd},{ }^{2} J_{\mathrm{HH}}=12.2\right.$, ${ }^{3} J_{\mathrm{HH}}=8.7$ and $\left.7.2 \mathrm{~Hz}, \mathrm{~S}-\mathrm{CHaHb}\right), 2.72\left(1 \mathrm{H}, \mathrm{ddd},{ }^{2} J_{\mathrm{HH}}=12.2,{ }^{3} J_{\mathrm{HH}}=9.2\right.$ and $\left.5.0 \mathrm{~Hz}, \mathrm{~S}-\mathrm{C} \underline{\mathrm{HaHb}}\right)$, $4.65\left(1 \mathrm{H}, \mathrm{dtd},{ }^{3} J_{\mathrm{HP}}=17.5,{ }^{3} J_{\mathrm{HH}}=10.4,10.4\right.$ and $\left.3.7 \mathrm{~Hz}, \mathrm{~N}-\mathrm{CH}-\mathrm{P}\right), 5.12\left(2 \mathrm{H}, \mathrm{s}, \mathrm{O}-\mathrm{CH}_{2}\right), 5.40$ $\left(1 \mathrm{H}, \mathrm{dd},{ }^{3} J_{\mathrm{HH}}=10.4\right.$ and $\left.{ }^{3} J_{\mathrm{HP}}=1.5 \mathrm{~Hz}, \mathrm{NH}\right), 7.08-7.34\left(15 \mathrm{H}_{\text {arom }}, \mathrm{m}, 15 \mathrm{CH}\right) .{ }^{13} \mathrm{C} \mathrm{NMR}(150.9$ $\left.\mathrm{MHz} ; \mathrm{CDCl}_{3}\right): \delta_{\mathrm{C}} 24.47\left(1 \mathrm{C}, \mathrm{d},{ }^{3} J_{\mathrm{CP}}=15.1 \mathrm{~Hz}, \mathrm{~S}-\mathrm{CH}_{2}\right), 30.68\left(1 \mathrm{C}, \mathrm{d},{ }^{2} J_{\mathrm{CP}}=4.8 \mathrm{~Hz}, \mathrm{C}-\underline{C H}_{2}-\mathrm{C}\right)$, $30.87\left(3 \mathrm{C}, \mathrm{C}\left(\underline{\mathrm{CH}}_{3}\right)_{3}\right), 42.41\left(1 \mathrm{C}, \underline{\mathrm{C}}\left(\mathrm{CH}_{3}\right)_{3}\right), 47.96\left(1 \mathrm{C}, \mathrm{d},{ }^{1} J_{\mathrm{CP}}=158.6 \mathrm{~Hz}, \mathrm{~N}-\mathrm{CH}-\mathrm{P}\right), 67.30(1 \mathrm{C}$, $\left.\mathrm{CH}_{2}-\mathrm{O}\right), 120.33\left(2 \mathrm{C}, \mathrm{d},{ }^{3} J_{\mathrm{CP}}=4.1 \mathrm{~Hz}\right.$, ortho- $\left.\mathrm{CH}_{\text {arom }}\right), 120.53\left(2 \mathrm{C}, \mathrm{d},{ }^{3} J_{\mathrm{CP}}=4.1 \mathrm{~Hz}\right.$, ortho$\left.\mathrm{CH}_{\text {arom }}\right), 125.26,125.41,128.11(2), 128.22,128.50(2), 129.66(2)$ and 129.79(2) (11C, $\left.\mathrm{CH}_{\text {arom }}\right)$, $136.02\left(1 \mathrm{C}, \mathrm{C}_{\text {arom }}\right), 149.87\left(1 \mathrm{C}, \mathrm{d},{ }^{2} J_{\mathrm{CP}}=9.7 \mathrm{~Hz}\right.$, ipso- $\left.\mathrm{C}_{\mathrm{arom}}\right), 150.12\left(1 \mathrm{C}, \mathrm{d},{ }^{3} J_{\mathrm{CP}}=9.8 \mathrm{~Hz}\right.$, ipso$\left.\mathrm{C}_{\text {arom }}\right), 155.82\left(1 \mathrm{C}, \mathrm{d},{ }^{3} J_{\mathrm{CP}}=6.1 \mathrm{~Hz}, \mathrm{~N}-\mathrm{CO}-\mathrm{O}\right)$. IR $\left(\mathrm{KBr}, v_{\max }, \mathrm{cm}^{-1}\right) 3287 \mathrm{~m}(\mathrm{NH}) ; 1540 \mathrm{~s}$ $(\mathrm{CONH}) ; 1723$ vs $(\mathrm{C}=\mathrm{O}) ; 1248 \mathrm{~s}, 1229 \mathrm{~s}(\mathrm{P}=\mathrm{O}) ; 1218 \mathrm{~s}, 1206 \mathrm{~s}, 1189 \mathrm{~s}, 1163 \mathrm{~s}(\mathrm{Ph}-\mathrm{OP}) ; 950 \mathrm{vs}$ (P-OPh); 1593 m, 1492 vs, 1456 m, 1027 m, 765 s, 689 m, 525 m (ring); 2960 m (CH3). HRMS (ESI) calc for $\mathrm{C}_{27} \mathrm{H}_{33} \mathrm{O}_{5} \mathrm{NPS}[\mathrm{M}+1]^{+}$514.18116; found: 514.18086 .

Diphenyl [(R,S)-1-(benzyloxycarbonylamino)-3-(benzylsulfanyl)propyl]phosphonate (4c). Using the procedure as outlined for $\mathbf{4 a}$, compound $\mathbf{4 c}$ was prepared from benzyl carbamate (21.2 g; $0.14 \mathrm{~mol}$ ), triphenylphosphite (43.4 g; $0.14 \mathrm{~mol}$ ) and 3-(benzylsulfanyl)propanal (1c) (27 g; $0.15 \mathrm{~mol})$. The residue was triturated at $-20^{\circ} \mathrm{C}$ using a mixture of ethylacetate-petroleum ether to afford a waxy material. Yield $42 \mathrm{~g}(55 \%) . \mathrm{R}_{\mathrm{f}}=0.30(\mathrm{~S} 1) .{ }^{1} \mathrm{H} \mathrm{NMR}\left(600 \mathrm{MHz}, \mathrm{CDCl}_{3}\right): \delta_{\mathrm{H}} 1.96$ $(1 \mathrm{H}, \mathrm{m}, \mathrm{C}-\mathrm{CHaHb}-\mathrm{C}), 2.25(1 \mathrm{H}, \mathrm{m}, \mathrm{C}-\mathrm{CHaHb}-\mathrm{C}), 2.51\left(1 \mathrm{H}, \mathrm{ddd},{ }^{2} J_{\mathrm{HH}}=13.5,{ }^{3} \mathrm{~J}_{\mathrm{HH}}=8.6\right.$ and 7.3 $\mathrm{Hz}, \mathrm{S}-\mathrm{C} \underline{\mathrm{HaHb}}), 2.59\left(1 \mathrm{H}, \mathrm{ddd},{ }^{2} J_{\mathrm{HH}}=13.5,{ }^{3} J_{\mathrm{HH}}=8.6\right.$ and $\left.4.7 \mathrm{~Hz}, \mathrm{~S}-\mathrm{CHaHb}\right), 4.61\left(1 \mathrm{H}, \mathrm{dtd},{ }^{3} J_{\mathrm{HP}}\right.$ $=17.5,{ }^{3} J_{\mathrm{HH}}=10.5,10.5$ and $\left.3.6 \mathrm{~Hz}, \mathrm{~N}-\mathrm{CH}-\mathrm{P}\right), 5.09\left(1 \mathrm{H}, \mathrm{d},{ }^{2} J_{\mathrm{HH}}=12.0 \mathrm{~Hz}, \mathrm{CHaHb}-\mathrm{O}\right), 5.11$ $\left(1 \mathrm{H}, \mathrm{d},{ }^{2} J_{\mathrm{HH}}=12.0 \mathrm{~Hz}, \mathrm{CHaHb}-\mathrm{O}\right), 5.25\left(1 \mathrm{H}, \mathrm{dd},{ }^{3} J_{\mathrm{HH}}=10.2\right.$ and $\left.{ }^{3} J_{\mathrm{HP}}=1.5 \mathrm{~Hz}, \mathrm{NH}\right), 7.06-7.34$ $\left(20 \mathrm{H}_{\text {arom }}, \mathrm{m}, 20 \mathrm{CH}\right) .{ }^{13} \mathrm{C} \mathrm{NMR}\left(150.9 \mathrm{MHz} ; \mathrm{CDCl}_{3}\right): \delta_{\mathrm{C}} 27.27\left(1 \mathrm{C}, \mathrm{d},{ }^{3} J_{\mathrm{CP}}=15.1 \mathrm{~Hz}, \mathrm{~S}-\mathrm{CH}_{2}\right)$, $30.02\left(1 \mathrm{C}, \mathrm{d},{ }^{2} J_{\mathrm{CP}}=4.7 \mathrm{~Hz}, \mathrm{C}-\underline{C H}_{2}-\mathrm{C}\right), 36.11\left(1 \mathrm{C}, \mathrm{S}-\mathrm{CH}_{2}\right), 47.64\left(1 \mathrm{C}, \mathrm{d},{ }^{1} J_{\mathrm{CP}}=158.7 \mathrm{~Hz}, \mathrm{~N}-\mathrm{CH}-\right.$ P), $67.38\left(1 \mathrm{C}, \mathrm{CH}_{2}-\mathrm{O}\right), 120.32\left(2 \mathrm{C}, \mathrm{d},{ }^{3} J_{\mathrm{CP}}=4.2 \mathrm{~Hz}\right.$, ortho- $\left.\mathrm{CH}_{\text {arom }}\right), 120.54\left(2 \mathrm{C}, \mathrm{d},{ }^{3} J_{\mathrm{CP}}=4.0 \mathrm{~Hz}\right.$, ortho- $\mathrm{CH}_{\text {arom }}$ ), 125.29, 125.46, 127.03, 128.15(2), 128.27, 128.50(2), 128.53, 128.83(3), $129.69(2)$ and 129.82(2) (16C, $\left.\mathrm{CH}_{\text {arom }}\right), 136.00$ (1C, ipso- $\left.\mathrm{C}_{\text {arom }}\right), 137.96$ (1C, ipso- $\left.\mathrm{C}_{\text {arom }}\right), 149.91$ $\left(1 \mathrm{C}, \mathrm{d},{ }^{2} J_{\mathrm{CP}}=9.7 \mathrm{~Hz}\right.$, ipso-C $\left.\mathrm{C}_{\mathrm{arom}}\right), 150.14\left(1 \mathrm{C}, \mathrm{d},{ }^{3} J_{\mathrm{CP}}=10.0 \mathrm{~Hz}\right.$, ipso- $\left.\mathrm{C}_{\mathrm{arom}}\right), 155.81\left(1 \mathrm{C}, \mathrm{d},{ }^{3} J_{\mathrm{CP}}\right.$ $=6.2 \mathrm{~Hz}, \mathrm{~N}-\mathrm{CO}-\mathrm{O})$. IR $\left(\mathrm{CCl}_{4}, v_{\max }, \mathrm{cm}^{-1}\right) 3432 \mathrm{w}, 3261 \mathrm{w}(\mathrm{NH}) ; 1535 \mathrm{~m}, 1491 \mathrm{vs}(\mathrm{CONH})$; 1727 vs $(\mathrm{C}=\mathrm{O}) ; 1265 \mathrm{~s}(\mathrm{P}=\mathrm{O}) ; 1211$ vs, 1189 vs, $1163 \mathrm{~s}(\mathrm{Ph}-\mathrm{OP}) ; 945$ vs $(\mathrm{P}-\mathrm{OPh}) ; 1593 \mathrm{~m}, 1455$ m, $1027 \mathrm{~s}, 1481 \mathrm{~s}, 765 \mathrm{~m}, 697 \mathrm{~s}, 689 \mathrm{~s}$ (ring). HRMS (ESI) calc for $\mathrm{C}_{30} \mathrm{H}_{30} \mathrm{O}_{5} \mathrm{NNaPS}[\mathrm{M}+\mathrm{Na}]^{+}$ 570.14745; found: 570.14743 . 
[3-(Phenylsulfanyl)-1-(R,S)-(diphenoxyphosphoryl)propan-1-aminium] bromide

(5a).

Phosphonate 4a (26.5 g; $49.7 \mathrm{mmol})$ was suspended in $50 \mathrm{ml}$ of $30 \% \mathrm{HBr} / \mathrm{AcOH}$. The reaction mixture was stirred and the solid material disappeared after 30 minutes accompanied by the extensive evolution of $\mathrm{CO}_{2}$. After standing for 2 hours, crystals of the hydrobromide had formed. They were removed by filtration and washed with diethyl ether. The pure product was obtained by recrystallization from a mixture of methanol-diethyl ether. Yield $19.5 \mathrm{~g}(82 \%)$. White solid, mp 168-170 ${ }^{\circ} \mathrm{C} . \mathrm{R}_{\mathrm{f}}=0.77$ (S4). ${ }^{1} \mathrm{H}$ NMR $\left(600 \mathrm{MHz}\right.$; $\left.\mathrm{d}_{6} \mathrm{DMSO}\right): \delta_{\mathrm{H}} 2.23(1 \mathrm{H}, \mathrm{m}, \mathrm{C}-\mathrm{CHaHb}-\mathrm{C})$, $2.27(1 \mathrm{H}, \mathrm{m}, \mathrm{C}-\mathrm{CHaHb}-\mathrm{C}), 3.24\left(1 \mathrm{H}, \mathrm{ddd},{ }^{2} J_{\mathrm{HH}}=13.9,{ }^{3} J_{\mathrm{HH}}=9.2\right.$ and $\left.6.7 \mathrm{~Hz}, \mathrm{~S}-\mathrm{CHaHb}\right), 3.33$ $\left(1 \mathrm{H}, \mathrm{ddd},{ }^{2} J_{\mathrm{HH}}=13.9,{ }^{3} J_{\mathrm{HH}}=9.3\right.$ and $\left.5.5 \mathrm{~Hz}, \mathrm{~S}-\mathrm{C} \underline{\mathrm{HaHb}}\right), 4.36\left(1 \mathrm{H}, \mathrm{ddd},{ }^{3} J_{\mathrm{HP}}=14.2,{ }^{3} J_{\mathrm{HH}}=7.9\right.$ and $5.8 \mathrm{~Hz}, \mathrm{~N}-\mathrm{CH}-\mathrm{P}), 7.16-7.43\left(15 \mathrm{H}_{\text {arom }}, \mathrm{m}, 15 \mathrm{CH}\right), 8.94\left(3 \mathrm{H}, \mathrm{bs}, \mathrm{NH}_{3}\right) .{ }^{13} \mathrm{C}$ NMR $(150.9$ MHz; $\left.\mathrm{d}_{6} \mathrm{DMSO}\right): \delta_{\mathrm{C}} 28.18\left(1 \mathrm{C}, \mathrm{S}-\mathrm{CH}_{2}\right), 28.21\left(1 \mathrm{C}, \mathrm{d},{ }^{2} J_{\mathrm{CP}}=9.8 \mathrm{~Hz}, \mathrm{C}-\underline{\mathrm{CH}}_{2}-\mathrm{C}\right), 45.66(1 \mathrm{C}, \mathrm{d}$, $\left.{ }^{1} J_{\mathrm{CP}}=156.7 \mathrm{~Hz}, \mathrm{~N}-\mathrm{CH}-\mathrm{P}\right), 120.72\left(2 \mathrm{C}, \mathrm{d},{ }^{3} J_{\mathrm{CP}}=4.4 \mathrm{~Hz}\right.$, ortho- $\left.\mathrm{CH}_{\text {arom }}\right), 120.74\left(2 \mathrm{C}, \mathrm{d},{ }^{3} J_{\mathrm{CP}}=4.2\right.$ $\mathrm{Hz}$, ortho $\left.-\mathrm{CH}_{\text {arom }}\right), 126.15,126.19,126.39,128.71(2), 129.47(2), 130.32(2)$ and 130.36(2) (11C, $\left.\mathrm{CH}_{\text {arom }}\right), 134.82\left(1 \mathrm{C}\right.$, ipso- $\left.\mathrm{C}_{\text {arom }}\right), 149.34\left(1 \mathrm{C}, \mathrm{d},{ }^{2} J_{\mathrm{CP}}=3.6 \mathrm{~Hz}\right.$, ipso- $\left.\mathrm{C}_{\text {arom }}\right), 149.40\left(1 \mathrm{C}, \mathrm{d},{ }^{3} J_{\mathrm{CP}}=\right.$ $3.5 \mathrm{~Hz}$, ipso-C $\left.\mathrm{C}_{\text {arom }}\right)$. IR ( $\left.\mathrm{KBr}, v_{\max }, \mathrm{cm}^{-1}\right) 3100-2400 \mathrm{vs}+\mathrm{vbr}, 1513 \mathrm{~s}\left(\mathrm{NH}_{3}{ }^{+}\right) ; 1252 \mathrm{~m}, 1234 \mathrm{~m}$ $(\mathrm{P}=\mathrm{O}) ; 1210 \mathrm{~s}, 1182$ vs (Ph-OP); 960 vs, 941 vs $(\mathrm{P}-\mathrm{OPh}) ; 1588 \mathrm{~s}, 1487$ vs, $1161 \mathrm{~s}, 1069$ m, 1024 m, 769 s, $740 \mathrm{~s}, 690 \mathrm{~s}$ (ring). HRMS (ESI) calc for $\mathrm{C}_{21} \mathrm{H}_{23} \mathrm{O}_{3} \mathrm{NPS}[\mathrm{M}+1]^{+} 400.11308$; found: 400.11339 .

[3-(tert-Butylsulfanyl)-1-( $R, S)$-(diphenoxyphosphoryl)propan-1-aminium] bromide $(5 \mathrm{~b})$. The salt $\mathbf{5 b}$ was prepared in the same manner as $\mathbf{5 a}$ by the reaction of phosphonate $\mathbf{4 b}(30 \mathrm{~g} ; 58.4$ $\mathrm{mmol}$ ) with $50 \mathrm{ml}$ of $30 \% \mathrm{HBr} / \mathrm{AcOH}$. Yield $21 \mathrm{~g}(78 \%)$. White solid, $\mathrm{mp} 154-155^{\circ} \mathrm{C}$. $\mathrm{R}_{\mathrm{f}}=0.82$ (S4). ${ }^{1} \mathrm{H}$ NMR (600 MHz; d $\left.\mathrm{d}_{6} \mathrm{DMSO}\right): \delta_{\mathrm{H}} 1.25\left(9 \mathrm{H}, \mathrm{s}, \mathrm{C}\left(\mathrm{CH}_{3}\right)_{3}\right), 2.12(1 \mathrm{H}, \mathrm{m}, \mathrm{C}-\mathrm{CHaHb}-\mathrm{C}), 2.22$ $(1 \mathrm{H}, \mathrm{m}, \mathrm{C}-\mathrm{CHaHb}-\mathrm{C}), 2.75\left(1 \mathrm{H}, \mathrm{ddd},{ }^{2} J_{\mathrm{HH}}=12.9,{ }^{3} J_{\mathrm{HH}}=10.3\right.$ and $\left.5.8 \mathrm{~Hz}, \mathrm{~S}-\mathrm{CHaHb}\right), 2.81(1 \mathrm{H}$, ddd, ${ }^{2} J_{\mathrm{HH}}=12.9,{ }^{3} J_{\mathrm{HH}}=10.4$ and $\left.5.7 \mathrm{~Hz}, \mathrm{~S}-\mathrm{CHaHb}\right), 4.26\left(1 \mathrm{H}, \mathrm{ddd},{ }^{3} J_{\mathrm{HP}}=14.1,{ }^{3} J_{\mathrm{HH}}=7.4\right.$ and $6.0 \mathrm{~Hz}, \mathrm{~N}-\mathrm{CH}-\mathrm{P}), 7.20\left(4 \mathrm{H}_{\text {arom }}, \mathrm{m}, 4 \mathrm{CH}\right), 7.27\left(2 \mathrm{H}_{\text {arom }}, \mathrm{m}, 2 \mathrm{CH}\right), 7.42\left(4 \mathrm{H}_{\text {arom }}, \mathrm{m}, 4 \mathrm{CH}\right) .{ }^{13} \mathrm{C}$

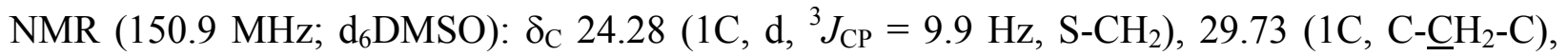
$31.19\left(3 \mathrm{C}, \mathrm{C}\left(\underline{\mathrm{CH}}_{3}\right)_{3}\right), 43.08\left(1 \mathrm{C}, \underline{\mathrm{C}}\left(\mathrm{CH}_{3}\right)_{3}\right), 46.24\left(1 \mathrm{C}, \mathrm{d},{ }^{1} J_{\mathrm{CP}}=156.2 \mathrm{~Hz}, \mathrm{~N}-\mathrm{CH}-\mathrm{P}\right), 120.93(2 \mathrm{C}$, $\mathrm{d},{ }^{3} J_{\mathrm{CP}}=4.0 \mathrm{~Hz}$, ortho- $\left.\mathrm{CH}_{\text {arom }}\right), 120.98\left(2 \mathrm{C}, \mathrm{d},{ }^{3} J_{\mathrm{CP}}=4.0 \mathrm{~Hz}\right.$, ortho- $\left.\mathrm{CH}_{\text {arom }}\right), 126.52(2)$, 130.64(2) and 130.66(2) (6C, $\left.\mathrm{CH}_{\text {arom }}\right), 149.63$ (2C, d, ${ }^{2} J_{\mathrm{CP}}=9.5 \mathrm{~Hz}$, ipso- $\left.\mathrm{C}_{\text {arom }}\right)$. IR ( $\mathrm{KBr}, v_{\max }$,

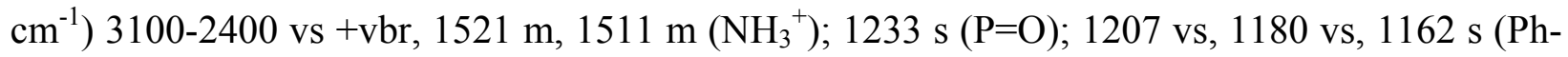
OP); 954 vs (P-OPh); 1589 s, 1489 vs, 1456 m, 1025 m, 1009 m, 910 m, 770 s, 689 s (ring); 2960 $\mathrm{s}\left(\mathrm{CH}_{3}\right)$. HRMS (ESI) calc for $\mathrm{C}_{19} \mathrm{H}_{27} \mathrm{O}_{3} \mathrm{NPS}[\mathrm{M}+1]^{+} 380.14438$; found: 380.14442 .

[3-(benzylsulfanyl)-1-(R,S)-(diphenoxyphosphoryl)propan-1-aminium] bromide (5c). The salt $5 \mathbf{c}$ was prepared in the same manner as $\mathbf{5 a}$ by the reaction of phosphonate $4 \mathbf{c}(30 \mathrm{~g} ; 54.8$ $\mathrm{mmol}$ ) with $50 \mathrm{ml}$ of $30 \%$ of $\mathrm{HBr} / \mathrm{AcOH}$. Yield $20.3 \mathrm{~g} \mathrm{(75 \% ).} \mathrm{White} \mathrm{solid,} \mathrm{mp} \mathrm{197-198}{ }^{\circ} \mathrm{C} . \mathrm{R}_{\mathrm{f}}=$ 0.80 (S4). ${ }^{1} \mathrm{H}$ NMR $\left(600 \mathrm{MHz} ; \mathrm{d}_{6} \mathrm{DMSO}\right): \delta_{\mathrm{H}} 2.29\left(2 \mathrm{H}, \mathrm{m}, \mathrm{C}-\mathrm{C}_{2}-\mathrm{C}\right), 2.75\left(2 \mathrm{H}, \mathrm{m}, \mathrm{S}-\mathrm{C}_{2}\right), 4.29$ $(1 \mathrm{H}, \mathrm{m}, \mathrm{N}-\mathrm{CH}-\mathrm{P}), 3.76\left(1 \mathrm{H}, \mathrm{d},{ }^{2} J_{\mathrm{HH}}=13.2 \mathrm{~Hz}, \mathrm{~S}-\mathrm{CHaHb}\right), 3.78\left(1 \mathrm{H}, \mathrm{d},{ }^{2} J_{\mathrm{HH}}=13.2 \mathrm{~Hz}, \mathrm{~S}-\right.$ $\mathrm{CHaHb}), 7.22\left(5 \mathrm{H}_{\text {arom }}, \mathrm{m}, 5 \mathrm{CH}\right), 7.26\left(2 \mathrm{H}_{\text {arom }}, \mathrm{m}, 2 \mathrm{CH}\right), 7.29\left(2 \mathrm{H}_{\text {arom }}, \mathrm{m}, 2 \mathrm{CH}\right), 7.32\left(2 \mathrm{H}_{\text {arom }}, \mathrm{m}\right.$, $2 \mathrm{CH}), 7.42\left(4 \mathrm{H}_{\text {arom }}, \mathrm{m}, 4 \mathrm{CH}\right), 8.99\left(3 \mathrm{H}, \mathrm{bs}, \mathrm{NH}_{3}{ }^{+}\right) .{ }^{13} \mathrm{C} \mathrm{NMR}\left(150.9 \mathrm{MHz} ; \mathrm{d}_{6} \mathrm{DMSO}\right): \delta_{\mathrm{C}} 26.91$ $\left(1 \mathrm{C}, \mathrm{d},{ }^{3} J_{\mathrm{CP}}=9.9 \mathrm{~Hz}, \mathrm{~S}-\mathrm{CH}_{2}\right), 28.29\left(1 \mathrm{C}, \mathrm{d},{ }^{2} J_{\mathrm{CP}}=1.3 \mathrm{~Hz}, \mathrm{C}-\underline{\mathrm{CH}}_{2}-\mathrm{C}\right), 34.88\left(1 \mathrm{C}, \mathrm{S}-\mathrm{CH}_{2}\right), 45.65$ 
$\left(1 \mathrm{C}, \mathrm{d},{ }^{1} J_{\mathrm{CP}}=156.5 \mathrm{~Hz}, \mathrm{~N}-\mathrm{CH}-\mathrm{P}\right), 120.68\left(2 \mathrm{C}, \mathrm{d},{ }^{3} J_{\mathrm{CP}}=4.1 \mathrm{~Hz}\right.$, ortho- $\left.\mathrm{CH}_{\mathrm{arom}}\right), 120.75(2 \mathrm{C}, \mathrm{d}$, ${ }^{3} J_{\mathrm{CP}}=4.1 \mathrm{~Hz}$, ortho- $\left.\mathrm{CH}_{\text {arom }}\right), 126.05(2), 127.02,128.57(2), 128.98(2), 130.23(2)$ and 130.26(2) (11C, $\mathrm{CH}_{\text {arom }}$ ), 138.51 (1C, ipso- $\left.\mathrm{C}_{\text {arom }}\right), 149.41\left(\mathrm{~d},{ }^{2} J_{\mathrm{CP}}=9.5 \mathrm{~Hz}\right.$, ipso- $\left.\mathrm{C}_{\text {arom }}\right), 149.44\left(1 \mathrm{C}, \mathrm{d},{ }^{2} J_{\mathrm{CP}}\right.$ $=9.5 \mathrm{~Hz}$, ipso- $\left.\mathrm{C}_{\text {arom }}\right)$. IR $\left(\mathrm{KBr}, v_{\max }, \mathrm{cm}^{-1}\right) 3100-2400 \mathrm{vs}+\mathrm{vbr}, 1521 \mathrm{~s}\left(\mathrm{NH}_{3}{ }^{+}\right) ; 1249 \mathrm{~s}(\mathrm{P}=\mathrm{O})$; 1200 vs, 1175 vs, 1160 vs (Ph-OP); 957 vs, 941 vs (P-OPh); 1587 s, 1486 vs, 1454 s, 1024 m, 1010 s, 770 s, 689 s (ring). HRMS (ESI) calc for $\mathrm{C}_{22} \mathrm{H}_{25} \mathrm{O}_{3} \mathrm{NPS}[\mathrm{M}+1]^{+}$414.12873; found: 414.12866 .

Diphenyl [(R,S)-1-(tert-butoxycarbonylamino)-3-(phenylsulfanyl)propyl]phosphonate (6a). TEA (2.9 g; $28.3 \mathrm{mmol})$ was added to a suspension of salt 5a (13.6 g; $28.3 \mathrm{mmol})$ in $250 \mathrm{ml}$ of dichloromethane. As soon as the solution clarified (approximately 10 minutes), the reaction flask was immersed in an ice bath, and $\mathrm{Boc}_{2} \mathrm{O}(6.8 \mathrm{~g} ; 31.1 \mathrm{mmol})$ in $50 \mathrm{ml}$ of DCM was added dropwise. After stirring for one hour at $0{ }^{\circ} \mathrm{C}$ and then overnight at $\mathrm{rt}$, the solvent was removed by rotary evaporator, and the oily residue was purified by flash chromatography on silica gel using a linear gradient of ethyl acetate in toluene as the eluant. The yield from the reaction was $10.5 \mathrm{~g}$ $(74 \%)$.

Alternatively, compound 6a was prepared according to procedure described by Van der Veken et al. $^{15}$ Aldehyde $1 \mathrm{a}(2 \mathrm{~g} ; 12 \mathrm{mmol})$, tert-butyl carbamate ${ }^{20}(1.2 \mathrm{~g} ; 10 \mathrm{mmol})$ and triphenyl phosphite $(3.1 \mathrm{~g} ; 10 \mathrm{mmol})$ were dissolved in $25 \mathrm{ml}$ of dry dichloromethane. $\mathrm{TiCl}_{4}(0.1 \mathrm{eq}, 0.1 \mathrm{M}$ solution) was added in one portion. The reaction mixture turned dark red and a mild reflux of the solvent was observed due to the exothermic nature of the reaction. After stirring for $24 \mathrm{hr}$ at $\mathrm{rt}$, the dichloromethane was evaporated in vacuo, and the residue was purified twice by flash chromatography on silica gel using a linear gradient of ethyl acetate in toluene as the eluant. Yield $2.6 \mathrm{~g}(52 \%)$. White solid, mp $72-74{ }^{\circ} \mathrm{C} . \mathrm{R}_{\mathrm{f}}=0.47(\mathrm{~S} 6) .{ }^{1} \mathrm{H} \mathrm{NMR}\left(600 \mathrm{MHz} ; \mathrm{CDCl}_{3}\right): \delta_{\mathrm{H}}$ $1.45\left(9 \mathrm{H}, \mathrm{s}, \mathrm{C}\left(\mathrm{CH}_{3}\right)_{3}\right), 2.03(1 \mathrm{H}, \mathrm{m}, \mathrm{C}-\mathrm{CHaHb}-\mathrm{C}), 2.32(1 \mathrm{H}, \mathrm{m}, \mathrm{C}-\mathrm{CHaHb}-\mathrm{C}), 3.01$ (1H, ddd, ${ }^{2} J_{\mathrm{HH}}=13.5,{ }^{3} J_{\mathrm{HH}}=8.8$ and $\left.7.0 \mathrm{~Hz}, \mathrm{~S}-\mathrm{C} \underline{\mathrm{HaHb}}\right), 3.13\left(1 \mathrm{H}, \mathrm{ddd},{ }^{2} J_{\mathrm{HH}}=13.5,{ }^{3} J_{\mathrm{HH}}=9.2\right.$ and 4.7 $\mathrm{Hz}, \mathrm{S}-\mathrm{C} \underline{\mathrm{HaHb}}), 4.62\left(1 \mathrm{H}, \mathrm{dtd},{ }^{3} J_{\mathrm{HP}}=18.2,{ }^{3} J_{\mathrm{HH}}=10.4,10.4\right.$ and $\left.3.8 \mathrm{~Hz}, \mathrm{~N}-\mathrm{CH}-\mathrm{P}\right), 7.12\left(4 \mathrm{H}_{\text {arom}}\right.$, $\mathrm{m}, 4 \mathrm{CH}), 7.17\left(2 \mathrm{H}_{\text {arom }}, \mathrm{m}, 2 \mathrm{CH}\right), 7.19\left(1 \mathrm{H}_{\text {arom }}, \mathrm{m}, 1 \mathrm{CH}\right), 7.28\left(2 \mathrm{H}_{\text {arom }}, \mathrm{m}, 2 \mathrm{CH}\right), 7.30\left(4 \mathrm{H}_{\text {arom }}, \mathrm{m}\right.$, $4 \mathrm{CH}), 7.36\left(2 \mathrm{H}_{\text {arom }}, \mathrm{m}, 2 \mathrm{CH}\right),{ }^{13} \mathrm{C} \mathrm{NMR}\left(150.9 \mathrm{MHz} ; \mathrm{CDCl}_{3}\right): \delta_{\mathrm{C}} 28.24\left(3 \mathrm{C}, \mathrm{C}\left(\underline{\mathrm{CH}}_{3}\right)_{3}\right), 30.27$ $\left(1 \mathrm{C}, \mathrm{C}-\underline{\mathrm{CH}}_{2}-\mathrm{C}\right), 30.23\left(1 \mathrm{C}, \mathrm{d},{ }^{3} J_{\mathrm{CP}}=19.5 \mathrm{~Hz}, \mathrm{~S}-\mathrm{CH}_{2}\right), 47.40\left(1 \mathrm{C}, \mathrm{d},{ }^{1} J_{\mathrm{CP}}=158.3 \mathrm{~Hz}, \mathrm{~N}-\mathrm{CH}-\mathrm{P}\right)$, $80.61\left(1 \mathrm{C}, \underline{\mathrm{C}}\left(\mathrm{CH}_{3}\right)_{3}\right), 120.38\left(2 \mathrm{C}, \mathrm{d},{ }^{3} J_{\mathrm{CP}}=4.1 \mathrm{~Hz}\right.$, ortho- $\left.\mathrm{CH}_{\text {arom }}\right), 120.50\left(2 \mathrm{C}, \mathrm{d},{ }^{3} J_{\mathrm{CP}}=4.1 \mathrm{~Hz}\right.$, ortho- $\left.\mathrm{CH}_{\text {arom }}\right), 125.32,125.43,126.42,129.00(2), 129.73(2), 129.83(2)$ and 129.92(2) (11C, $\mathrm{CH}_{\text {arom }}$ ), 135.31 (1C, ipso- $\left.\mathrm{C}_{\text {arom }}\right), 149.89\left(1 \mathrm{C}, \mathrm{d},{ }^{2} J_{\mathrm{CP}}=9.7 \mathrm{~Hz}\right.$, ipso- $\left.\mathrm{C}_{\text {arom }}\right), 150.20\left(1 \mathrm{C}, \mathrm{d},{ }^{2} J_{\mathrm{CP}}=\right.$ $10.0 \mathrm{~Hz}$, ipso- $\left.\mathrm{C}_{\text {arom }}\right), 155.04\left(1 \mathrm{C}, \mathrm{d},{ }^{3} J_{\mathrm{CP}}=6.9 \mathrm{~Hz}, \mathrm{~N}-\mathrm{CO}-\mathrm{O}\right) . \mathrm{IR}\left(\mathrm{KBr}, v_{\max }, \mathrm{cm}^{-1}\right) 3406 \mathrm{~m}, 3273$ s $(\mathrm{NH}) ; 1526$ s $(\mathrm{CONH}) ; 1713$ vs $(\mathrm{C}=\mathrm{O}) ; 1246$ vs $(\mathrm{P}=\mathrm{O}) ; 1212$ vs, 1189 vs (Ph-OP); 943 vs (P$\mathrm{OPh}$ ); $1591 \mathrm{~s}, 1440 \mathrm{~s}, 1291 \mathrm{~s}, 1071 \mathrm{~m}, 1025 \mathrm{~s}, 766 \mathrm{~s}, 740 \mathrm{~s}, 690 \mathrm{vs}$ (ring); $1367 \mathrm{~s}\left(\mathrm{CH}_{3}\right), 1163 \mathrm{vs}$

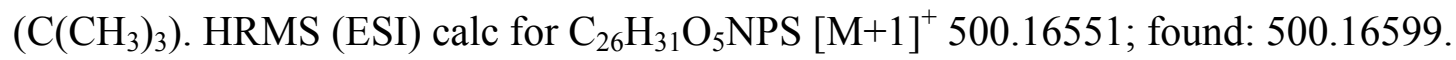

Diphenyl [(R,S)-1-(tert-butoxycarbonylamino)-3-(tert-butylsulfanyl)propyl]phosphonate (6b). Phosphonate 6b was prepared by the reaction of $\mathbf{5 b}(18.7 \mathrm{~g} ; 41 \mathrm{mmol})$, TEA (4.1 g; 41 $\mathrm{mmol})$ and $\mathrm{Boc}_{2} \mathrm{O}(10.7 \mathrm{~g} ; 49.2 \mathrm{mmol})$ as described above for $6 \mathbf{a}$. The yield was $12.6 \mathrm{~g}(65 \%)$. 
Phosphonate $\mathbf{6 b}$ was also synthesized using the alternate protocol for $\mathbf{6 a}$ by the reaction of aldehyde $\mathbf{1 b}$ (3.5 g; $24 \mathrm{mmol})$, tert-butyl carbamate ${ }^{20}$ (2.3 g; $\left.20 \mathrm{mmol}\right)$ and triphenyl phosphite (6.2 g; $20 \mathrm{mmol})$. Yield $5.1 \mathrm{~g}$ (54\%). Semisolid. $\mathrm{R}_{\mathrm{f}}=0.52$ (S2). ${ }^{1} \mathrm{H}$ NMR (600 MHz; $\left.\mathrm{d}_{6} \mathrm{DMSO}\right)$ : $\delta_{\mathrm{H}} 1.31\left(9 \mathrm{H}, \mathrm{s}, \mathrm{C}\left(\mathrm{CH}_{3}\right)_{3}\right), 1.44\left(9 \mathrm{H}, \mathrm{s}, \mathrm{C}\left(\mathrm{CH}_{3}\right)_{3}\right), 2.00(1 \mathrm{H}, \mathrm{m}, \mathrm{C}-\mathrm{CHaHb}-\mathrm{C}), 2.28(1 \mathrm{H}, \mathrm{m}, \mathrm{C}-$ CHa $\underline{\text { Hb}}-\mathrm{C}), 2.65\left(1 \mathrm{H}, \mathrm{ddd},{ }^{2} J_{\mathrm{HH}}=12.4,{ }^{3} J_{\mathrm{HH}}=9.2\right.$ and $\left.7.4 \mathrm{~Hz}, \mathrm{~S}-\mathrm{CHaHb}\right), 2.74\left(1 \mathrm{H}, \mathrm{ddd},{ }^{2} J_{\mathrm{HH}}=\right.$ $12.4,{ }^{3} J_{\mathrm{HH}}=9.5$ and $\left.4.8 \mathrm{~Hz}, \mathrm{~S}-\mathrm{CHaHb}\right), 4.59\left(1 \mathrm{H}, \mathrm{dtd},{ }^{3} J_{\mathrm{HP}}=17.6,{ }^{3} J_{\mathrm{HH}}=10.6,10.6\right.$ and $3.8 \mathrm{~Hz}$, $\mathrm{N}-\mathrm{CH}-\mathrm{P}), 4.96\left(1 \mathrm{H}, \mathrm{dd},{ }^{3} J_{\mathrm{HH}}=10.6\right.$ and $\left.{ }^{3} J_{\mathrm{HP}}=1.5 \mathrm{~Hz}, \mathrm{NH}\right), 7.13\left(2 \mathrm{H}_{\text {arom }}, \mathrm{m}, 2 \mathrm{CH}\right), 7.17\left(2 \mathrm{H}_{\text {arom, }}\right.$ $\mathrm{m}, 2 \mathrm{CH}), 7.19\left(2 \mathrm{H}_{\text {arom }}, \mathrm{m}, 2 \mathrm{CH}\right), 7.31\left(4 \mathrm{H}_{\text {arom }}, \mathrm{m}, 4 \mathrm{CH}\right) .{ }^{13} \mathrm{C}$ NMR $\left(150.9 \mathrm{MHz} ; \mathrm{d}_{6} \mathrm{DMSO}\right): \delta_{\mathrm{C}}$ $24.49\left(1 \mathrm{C}, \mathrm{d},{ }^{3} J_{\mathrm{CP}}=15.0 \mathrm{~Hz}, \mathrm{~S}-\mathrm{CH}_{2}\right), 28.22\left(3 \mathrm{C}, \mathrm{C}\left(\underline{\mathrm{CH}}_{3}\right)_{3}\right), 30.92\left(3 \mathrm{C}, \mathrm{C}\left(\underline{\mathrm{CH}}_{3}\right)_{3}\right), 42.39(1 \mathrm{C}, \mathrm{S}-$ $\left.\underline{\mathrm{C}}\left(\mathrm{CH}_{3}\right)_{3}\right), 47.28\left(1 \mathrm{C}, \mathrm{d},{ }^{1} J_{\mathrm{CP}}=158.2 \mathrm{~Hz}, \mathrm{~N}-\mathrm{CH}-\mathrm{P}\right), 80.46\left(1 \mathrm{C}, \mathrm{O}-\underline{\mathrm{C}}\left(\mathrm{CH}_{3}\right)_{3}\right), 120.40\left(2 \mathrm{C}, \mathrm{d},{ }^{3} J_{\mathrm{CP}}=\right.$ $4.1 \mathrm{~Hz}$, ortho- $\left.\mathrm{CH}_{\text {arom }}\right), 120.57\left(2 \mathrm{C}, \mathrm{d},{ }^{3} J_{\mathrm{CP}}=4.1 \mathrm{~Hz}\right.$, ortho- $\left.\mathrm{CH}_{\text {arom }}\right), 125.27,125.41,129.70(2)$ and $129.82(2)\left(6 \mathrm{C}, \mathrm{CH}_{\text {arom }}\right), 149.85\left(1 \mathrm{C}, \mathrm{d},{ }^{2} J_{\mathrm{CP}}=9.7 \mathrm{~Hz}\right.$, ipso- $\left.\mathrm{C}_{\mathrm{arom}}\right), 150.25\left(1 \mathrm{C}, \mathrm{d},{ }^{2} J_{\mathrm{CP}}=9.9\right.$ $\mathrm{Hz}$, ipso- $\left.\mathrm{C}_{\mathrm{arom}}\right), 155.03\left(1 \mathrm{C}, \mathrm{d},{ }^{3} J_{\mathrm{CP}}=6.5 \mathrm{~Hz}, \mathrm{~N}-\mathrm{CO}-\mathrm{O}\right)$. IR $\left(\mathrm{CCl}_{4}, v_{\max }, \mathrm{cm}^{-1}\right) 3436 \mathrm{w}, 3274 \mathrm{w}$ $(\mathrm{NH}) ; 1525 \mathrm{~m}, 1490$ vs $(\mathrm{CONH}) ; 1721$ vs $(\mathrm{C}=\mathrm{O}) ; 1242 \mathrm{~m}(\mathrm{P}=\mathrm{O}) ; 1213 \mathrm{~s}, 1190 \mathrm{vs}, 1163 \mathrm{vs}(\mathrm{Ph}-$ OP); 942 vs (P-OPh); 1594 m, 1457 m, 1026 m, 688 m., (ring); 2976 m, 1457 m, 1392 m, 1367 $\mathrm{m}\left(\mathrm{CH}_{3}\right)$. HRMS (ESI) calc for $\mathrm{C}_{24} \mathrm{H}_{34} \mathrm{O}_{5} \mathrm{NNaPS}[\mathrm{M}+\mathrm{Na}]^{+}$502.17875; found: 502.17873 .

Diphenyl [(R,S)-1-(tert-butoxycarbonylamino)-3-(benzylsulfanyl)propyl]phosphonate (6c). Phosphonate 6c was prepared by the reaction of $\mathbf{5 c}(20 \mathrm{~g} ; 40.1 \mathrm{mmol})$, TEA (4.1 g; $40.1 \mathrm{mmol})$ and $\mathrm{Boc}_{2} \mathrm{O}(9.6 \mathrm{~g} ; 44.1 \mathrm{mmol})$ as described above for $6 \mathbf{a}$. The yield was $15.5 \mathrm{~g}(75 \%)$.

Phosphonate 6c was also synthesized using the alternate protocol for $\mathbf{6 a}$ by the reaction of aldehyde 1c $(2.5 \mathrm{~g} ; 14 \mathrm{mmol})$, tert-butyl carbamate ${ }^{20}(1.5 \mathrm{~g} ; 12.8 \mathrm{mmol})$ and triphenyl phosphite (4 g; $12.8 \mathrm{mmol})$. Yield $3.2 \mathrm{~g}(49 \%) . \mathrm{R}_{\mathrm{f}}=0.45(\mathrm{~S} 2) .{ }^{1} \mathrm{H}$ NMR $\left(600 \mathrm{MHz} ; \mathrm{CDCl}_{3}\right): \delta_{\mathrm{H}} 1.43(9 \mathrm{H}$, $\left.\mathrm{s}, \mathrm{C}\left(\mathrm{CH}_{3}\right)_{3}\right), 1.94(1 \mathrm{H}, \mathrm{m}, \mathrm{C}-\mathrm{C} \underline{\mathrm{HaHb}}-\mathrm{C}), 2.25(1 \mathrm{H}, \mathrm{m}, \mathrm{C}-\mathrm{CHaHb}-\mathrm{C}), 2.52\left(1 \mathrm{H}, \mathrm{ddd},{ }^{2} J_{\mathrm{HH}}=13.2\right.$, ${ }^{3} J_{\mathrm{HH}}=9.0$ and $\left.7.0 \mathrm{~Hz}, \mathrm{~S}-\mathrm{CHaHb}\right), 2.61\left(1 \mathrm{H}, \mathrm{ddd},{ }^{2} J_{\mathrm{HH}}=13.2,{ }^{3} J_{\mathrm{HH}}=9.4\right.$ and $\left.4.6 \mathrm{~Hz}, \mathrm{~S}-\mathrm{C} \underline{\mathrm{HaHb}}\right)$, $3.71\left(2 \mathrm{H}, \mathrm{s}, \mathrm{S}-\mathrm{CH}_{2}\right), 4.54\left(1 \mathrm{H}, \mathrm{dtd},{ }^{3} J_{\mathrm{HP}}=13.0,{ }^{3} J_{\mathrm{HH}}=10.5,10.5\right.$ and $\left.3.5 \mathrm{~Hz}, \mathrm{~N}-\mathrm{CH}-\mathrm{P}\right), 4.87$ $\left(1 \mathrm{H}\right.$, bdd, ${ }^{3} J_{\mathrm{HH}}=10.5,{ }^{3} J_{\mathrm{HP}}=1.5,7.12-7.32\left(15 \mathrm{H}_{\text {arom }}, \mathrm{m}, 15 \mathrm{CH}\right) .{ }^{13} \mathrm{C} \mathrm{NMR}(150.9 \mathrm{MHz}$; $\left.\mathrm{CDCl}_{3}\right): \delta_{\mathrm{C}} 27.35\left(1 \mathrm{C}, \mathrm{d},{ }^{3} J_{\mathrm{CP}}=15.3 \mathrm{~Hz}, \mathrm{~S}-\mathrm{CH}_{2}\right), 28.24\left(3 \mathrm{C}, \mathrm{C}\left(\underline{\mathrm{CH}}_{3}\right)_{3}\right), 30.35\left(1 \mathrm{C}, \mathrm{d},{ }^{2} J_{\mathrm{CP}}=5.0\right.$ $\left.\mathrm{Hz}, \mathrm{C}-\underline{\mathrm{CH}}_{2}-\mathrm{C}\right), 36.18\left(1 \mathrm{C}, \mathrm{S}-\mathrm{CH}_{2}\right), 47.02\left(1 \mathrm{C}, \mathrm{d},{ }^{1} J_{\mathrm{CP}}=158.3 \mathrm{~Hz}, \mathrm{~N}-\mathrm{CH}-\mathrm{P}\right), 80.47(1 \mathrm{C}$, $\left.\underline{\mathrm{C}}\left(\mathrm{CH}_{3}\right)_{3}\right), 120.38\left(2 \mathrm{C}, \mathrm{d},{ }^{3} J_{\mathrm{CP}}=4.2 \mathrm{~Hz}\right.$, ortho- $\left.\mathrm{CH}_{\text {arom }}\right), 120.57\left(2 \mathrm{C}, \mathrm{d},{ }^{3} J_{\mathrm{CP}}=4.0 \mathrm{~Hz}\right.$, ortho$\left.\mathrm{CH}_{\text {arom }}\right), 125.27,125.42,127.03,128.52(2), 128.84(2), 129.71(2)$ and 129.83(2) (11C, $\left.\mathrm{CH}_{\text {arom }}\right)$, $138.06\left(1 \mathrm{C}\right.$, ipso-C $\left.\mathrm{C}_{\text {arom}}\right), 149.99\left(1 \mathrm{C}, \mathrm{d},{ }^{2} J_{\mathrm{CP}}=9.4 \mathrm{~Hz}\right.$, ipso- $\left.\mathrm{C}_{\text {arom }}\right), 150.28\left(1 \mathrm{C}, \mathrm{d},{ }^{2} J_{\mathrm{CP}}=9.4 \mathrm{~Hz}\right.$, ipso- $\left.\mathrm{C}_{\text {arom}}\right), 154.99\left(\mathrm{~d},{ }^{3} J_{\mathrm{CP}}=6.9 \mathrm{~Hz}, \mathrm{~N}-\mathrm{CO}-\mathrm{O}\right)$. IR $\left(\mathrm{CCl}_{4}, v_{\max }, \mathrm{cm}^{-1}\right) 3436 \mathrm{w}, 3273 \mathrm{~m} \mathrm{(NH)}$; $1525 \mathrm{~m}, 1491$ vs $(\mathrm{CONH}) ; 1719$ vs $(\mathrm{C}=\mathrm{O}) ; 1262$ vs $(\mathrm{P}=\mathrm{O}) ; 1213$ vs, 1190 vs, 1163 vs (Ph-OP); 943 vs (P-OPh); 1594 s, 1455 s, 1072 m, 1026 s, 698 s, 688 s (ring); 1367 s (CH $)$. HRMS (ESI) calc for $\mathrm{C}_{27} \mathrm{H}_{32} \mathrm{O}_{5} \mathrm{NNaPS}[\mathrm{M}+\mathrm{Na}]^{+}$536.16310; found: 536.16292 .

Di-tert-butyl [(R,S)-1-(tert-butoxycarbonylamino)-3-(phenylsulfanyl)propyl]phosphonate (7a). Potassium tert-butoxide ( $\mathrm{g} ; 62.4 \mathrm{mmol})$ was carefully added to a stirred solution of compound 6a (10 g; $18.7 \mathrm{mmol})$ dissolved in $100 \mathrm{ml}$ of anhydrous tert-butanol and $100 \mathrm{ml}$ of dry dioxane. The flask was equipped with a calcium dichloride tube and the reaction was allowed to proceed at rt overnight. The solvents were evaporated in vacuo, and the residue was taken up in 
$100 \mathrm{ml}$ of ethyl acetate and washed with $100 \mathrm{ml}$ of water and brine. The organic layer was separated, dried over $\mathrm{Na}_{2} \mathrm{SO}_{4}$, filtered and evaporated in vacuo. The crude product was purified by flash chromatography on silica gel using a linear gradient of ethyl acetate in toluene as the eluant. Yield $3.8 \mathrm{~g}(53 \%)$. Colorless oil, $\mathrm{R}_{\mathrm{f}}=0.79(\mathrm{~S} 3) .{ }^{1} \mathrm{H}$ NMR $\left(600 \mathrm{MHz} ; \mathrm{CDCl}_{3}\right): \delta_{\mathrm{H}} 1.455$ $\left(9 \mathrm{H}, \mathrm{s}, \mathrm{C}\left(\mathrm{CH}_{3}\right)_{3}\right), 1.475\left(9 \mathrm{H}, \mathrm{s}, \mathrm{C}\left(\mathrm{CH}_{3}\right)_{3}\right), 1.48\left(9 \mathrm{H}, \mathrm{s}, \mathrm{C}\left(\mathrm{CH}_{3}\right)_{3}\right), 1.75(1 \mathrm{H}, \mathrm{m}, \mathrm{C}-\mathrm{CHaHb}-\mathrm{C}), 2.19$ $(1 \mathrm{H}, \mathrm{m}, \mathrm{C}-\mathrm{CHaHb}-\mathrm{C}), 2.98\left(1 \mathrm{H}, \mathrm{ddd},{ }^{2} \mathrm{~J}_{\mathrm{HH}}=13.2,{ }^{3} J_{\mathrm{HH}}=9.5\right.$ and $\left.6.7 \mathrm{~Hz}, \mathrm{~S}-\mathrm{C} \underline{\mathrm{HaHb}}\right), 3.04(1 \mathrm{H}$, $\mathrm{ddd},{ }^{2} J_{\mathrm{HH}}=13.2,{ }^{3} J_{\mathrm{HH}}=9.7$ and $\left.5.0 \mathrm{~Hz}, \mathrm{~S}-\mathrm{CHaHb}\right), 3.98\left(1 \mathrm{H}, \mathrm{dtd},{ }^{3} J_{\mathrm{HP}}=17.2,{ }^{3} J_{\mathrm{HH}}=10.4,10.0\right.$ and $4.1 \mathrm{~Hz}, \mathrm{~N}-\mathrm{CH}-\mathrm{P}), 4.64\left(1 \mathrm{H}, \mathrm{dd},{ }^{3} J_{\mathrm{HH}}=10.4,{ }^{3} J_{\mathrm{HP}}=3.6, \mathrm{NH}\right), 7.17\left(1 \mathrm{H}_{\text {arom }}, \mathrm{m}, 1 \mathrm{CH}\right), 7.27$ $\left(2 \mathrm{H}_{\text {arom }}, \mathrm{m}, 2 \mathrm{CH}\right), 7.35\left(2 \mathrm{H}_{\text {arom }}, \mathrm{m}, 2 \mathrm{CH}\right) .{ }^{13} \mathrm{C} \mathrm{NMR}\left(150.9 \mathrm{MHz} ; \mathrm{CDCl}_{3}\right): \delta_{\mathrm{C}} 28.24(3 \mathrm{C}$, $\left.\mathrm{C}\left(\underline{\mathrm{CH}}_{3}\right)_{3}\right), 30.15\left(3 \mathrm{C}, \mathrm{d},{ }^{3} J_{\mathrm{CP}}=3.6, \mathrm{C}\left(\underline{\mathrm{CH}}_{3}\right)_{3}\right), 30.35\left(3 \mathrm{C}, \mathrm{d},{ }^{3} J_{\mathrm{CP}}=3.6, \mathrm{C}\left(\underline{\mathrm{CH}}_{3}\right)_{3}\right), 30.46(1 \mathrm{C}, \mathrm{S}-$ $\left.\mathrm{CH}_{2}\right), 31.13\left(1 \mathrm{C}, \mathrm{d},{ }^{2} J_{\mathrm{CP}}=3.7 \mathrm{~Hz}, \mathrm{C}-\underline{C H}_{2}-\mathrm{C}\right), 49.00\left(1 \mathrm{C}, \mathrm{d},{ }^{1} J_{\mathrm{CP}}=159.7 \mathrm{~Hz}, \mathrm{~N}-\mathrm{CH}-\mathrm{P}\right), 79.80$ $\left(1 \mathrm{C}, \underline{\mathrm{C}}\left(\mathrm{CH}_{3}\right)_{3}\right), 82.97\left(1 \mathrm{C}, \mathrm{d},{ }^{2} J_{\mathrm{CP}}=8.5 \mathrm{~Hz}, \underline{\mathrm{C}}\left(\mathrm{CH}_{3}\right)_{3}\right), 83.14\left(1 \mathrm{C}, \mathrm{d},{ }^{2} J_{\mathrm{CP}}=9.8 \mathrm{~Hz}, \underline{\mathrm{C}}\left(\mathrm{CH}_{3}\right)_{3}\right)$, 126.00 (1C, para- $\left.\mathrm{CH}_{\text {arom }}\right), 128.84$ (2C, meta- $\left.\mathrm{CH}_{\text {arom }}\right), 128.84$ (2C, ortho- $\left.\mathrm{CH}_{\text {arom }}\right), 135.99$ (1C, ipso- $\left.\mathrm{C}_{\text {arom }}\right), 155.35\left(1 \mathrm{C}, \mathrm{d},{ }^{3} J_{\mathrm{CP}}=6.5 \mathrm{~Hz}, \mathrm{~N}-\mathrm{CO}-\mathrm{O}\right)$. IR $\left(\mathrm{CCl}_{4}, v_{\max }, \mathrm{cm}^{-1}\right) 3449 \mathrm{w}, 3259 \mathrm{w}(\mathrm{NH})$; $1497 \mathrm{~s}(\mathrm{CONH}) ; 1718 \mathrm{~s}(\mathrm{C}=\mathrm{O}) ; 1248 \mathrm{~m}(\mathrm{P}=\mathrm{O}) ; 982 \mathrm{vs}(\mathrm{C}-\mathrm{OP}) ; 1482 \mathrm{~m}, 1440 \mathrm{~m}, 691 \mathrm{~m}$ (ring); $2981 \mathrm{~s}, 1369 \mathrm{~s}\left(\mathrm{CH}_{3}\right), 1170 \mathrm{~s}\left(\mathrm{C}\left(\mathrm{CH}_{3}\right)_{3}\right)$. HRMS (ESI) calc for $\mathrm{C}_{22} \mathrm{H}_{39} \mathrm{O}_{5} \mathrm{NPS}[\mathrm{M}+1]^{+} 460.22811$; found: 460.22836 .

Di-tert-butyl $[(R, S)$-1-(tert-butoxycarbonylamino)-3-(tert-butylsulfanyl)propyl]phosphonate (7b). Using the procedure outlined for $7 \mathbf{a}$, phosphonate $\mathbf{7 b}$ was prepared from compound $\mathbf{6 b}(8$ $\mathrm{g} ; 17 \mathrm{mmol})$ and potassium tert-butoxide $(7.6 \mathrm{~g} ; 68 \mathrm{mmol})$. Yield $4.3 \mathrm{~g}(59 \%)$. Semisolid, $\mathrm{R}_{\mathrm{f}}=$ $0.67(\mathrm{~S} 3) .{ }^{1} \mathrm{H}$ NMR $\left(600 \mathrm{MHz} ; \mathrm{CDCl}_{3}\right): \delta_{\mathrm{H}} 1.31\left(9 \mathrm{H}, \mathrm{s}, \mathrm{C}\left(\mathrm{CH}_{3}\right)_{3}\right), 1.45\left(9 \mathrm{H}, \mathrm{s}, \mathrm{C}\left(\mathrm{CH}_{3}\right)_{3}\right), 1.50$ $\left(9 \mathrm{H}, \mathrm{s}, \mathrm{C}\left(\mathrm{CH}_{3}\right)_{3}\right), 1.51\left(9 \mathrm{H}, \mathrm{s}, \mathrm{C}\left(\mathrm{CH}_{3}\right)_{3}\right), 1.68(1 \mathrm{H}, \mathrm{m}, \mathrm{C}-\mathrm{CHaHb}-\mathrm{C}), 2.15$ (1H, m, C-CHaHb-C), $2.59\left(1 \mathrm{H}, \mathrm{ddd},{ }^{2} J_{\mathrm{HH}}=12.0,{ }^{3} J_{\mathrm{HH}}=10.2\right.$ and $\left.6.5 \mathrm{~Hz}, \mathrm{~S}-\mathrm{CHaHb}\right), 2.65\left(1 \mathrm{H}, \mathrm{ddd},{ }^{2} J_{\mathrm{HH}}=12.0,{ }^{3} J_{\mathrm{HH}}\right.$ $=10.5$ and $4.8 \mathrm{~Hz}, \mathrm{~S}-\mathrm{CHaHb}), 3.93\left(1 \mathrm{H}, \mathrm{dtd},{ }^{3} J_{\mathrm{HP}}=16.6,{ }^{3} J_{\mathrm{HH}}=10.3,10.2\right.$ and $3.8 \mathrm{~Hz}, \mathrm{~N}-\mathrm{CH}-$ P), $4.62\left(1 \mathrm{H}, \mathrm{dd},{ }^{3} J_{\mathrm{HH}}=10.3,{ }^{3} J_{\mathrm{HP}}=3.2, \mathrm{NH}\right), 7.17\left(1 \mathrm{H}_{\text {arom }}, \mathrm{m}, 1 \mathrm{CH}\right), 7.27\left(2 \mathrm{H}_{\text {arom }}, \mathrm{m}, 2 \mathrm{CH}\right)$, $7.35\left(2 \mathrm{H}_{\text {arom }}, \mathrm{m}, 2 \mathrm{CH}\right) .{ }^{13} \mathrm{C} \mathrm{NMR}\left(150.9 \mathrm{MHz} ; \mathrm{CDCl}_{3}\right): \delta_{\mathrm{C}} 24.92\left(1 \mathrm{C}, \mathrm{d},{ }^{3} J_{\mathrm{CP}}=14.4, \mathrm{~S}-\mathrm{CH}_{2}\right)$, $28.30\left(3 \mathrm{C}, \mathrm{C}\left(\underline{\mathrm{CH}}_{3}\right)_{3}\right), 30.18\left(3 \mathrm{C}, \mathrm{d},{ }^{3} J_{\mathrm{CP}}=3.5, \mathrm{C}\left(\underline{\mathrm{CH}}_{3}\right)_{3}\right), 30.37\left(3 \mathrm{C}, \mathrm{d},{ }^{3} J_{\mathrm{CP}}=3.8, \mathrm{C}\left(\underline{\mathrm{CH}}_{3}\right)_{3}\right)$, $30.93\left(3 \mathrm{C}, \mathrm{C}\left(\underline{\mathrm{CH}}_{3}\right)_{3}\right), 31.73\left(1 \mathrm{C}, \mathrm{d},{ }^{2} J_{\mathrm{CP}}=3.6 \mathrm{~Hz}, \mathrm{C}-\underline{\mathrm{CH}}_{2}-\mathrm{C}\right), 42.14\left(1 \mathrm{C}, \underline{\mathrm{C}}\left(\mathrm{CH}_{3}\right)_{3}\right), 49.43(1 \mathrm{C}, \mathrm{d}$, $\left.{ }^{1} J_{\mathrm{CP}}=159.6 \mathrm{~Hz}, \mathrm{~N}-\mathrm{CH}-\mathrm{P}\right), 79.65\left(1 \mathrm{C}, \underline{\mathrm{C}}\left(\mathrm{CH}_{3}\right)_{3}\right), 82.86\left(1 \mathrm{C}, \mathrm{d},{ }^{2} J_{\mathrm{CP}}=8.4 \mathrm{~Hz}, \underline{\mathrm{C}}\left(\mathrm{CH}_{3}\right)_{3}\right), 83.03$ $\left(1 \mathrm{C}, \mathrm{d},{ }^{2} J_{\mathrm{CP}}=9.7 \mathrm{~Hz}, \underline{\mathrm{C}}\left(\mathrm{CH}_{3}\right)_{3}\right), 155.34\left(1 \mathrm{C}, \mathrm{d},{ }^{3} J_{\mathrm{CP}}=6.4 \mathrm{~Hz}, \mathrm{~N}-\mathrm{CO}-\mathrm{O}\right) . \mathrm{IR}\left(\mathrm{CCl}_{4}, v_{\max }, \mathrm{cm}^{-1}\right)$ 3450, $3256 \mathrm{w}(\mathrm{NH}) ; 1535 \mathrm{w}, 1497 \mathrm{~s}(\mathrm{CONH}) ; 1718 \mathrm{vs}(\mathrm{C}=\mathrm{O}) ; 1259 \mathrm{~s}, 1248 \mathrm{~s}(\mathrm{P}=\mathrm{O})$; 981 vs $(\mathrm{C}-$ OP); $2980 \mathrm{~s}, 1393 \mathrm{~m}, 1369 \mathrm{~s}\left(\mathrm{CH}_{3}\right) ; 1170$ vs $\left(\mathrm{C}\left(\mathrm{CH}_{3}\right)_{3}\right)$. HRMS (ESI) calc for $\mathrm{C}_{20} \mathrm{H}_{42} \mathrm{O}_{5} \mathrm{NNaPS}$ $[\mathrm{M}+\mathrm{Na}]^{+}$462.24135; found: 462.24132 .

Di-tert-butyl [(R,S)-1-(tert-butoxycarbonylamino)-3-(benzylsulfanyl)propyl]phosphonate (7c). Using the procedure outlined for 7a, phosphonate 7c was prepared from $6 \mathbf{c}(3.8 \mathrm{~g} ; 7.4$ $\mathrm{mmol})$ and potassium tert-butoxide $(3.3 \mathrm{~g} ; 29.6 \mathrm{mmol})$. Yield $2.1 \mathrm{~g}(60 \%)$. Colorless oil, $\mathrm{R}_{\mathrm{f}}=0.2$ (S2). ${ }^{1} \mathrm{H}$ NMR $\left(600 \mathrm{MHz} ; \mathrm{CDCl}_{3}\right): \delta_{\mathrm{H}} 1.44\left(9 \mathrm{H}, \mathrm{s}, \mathrm{C}\left(\mathrm{CH}_{3}\right)_{3}\right), 1.49\left(9 \mathrm{H}, \mathrm{s}, \mathrm{C}\left(\mathrm{CH}_{3}\right)_{3}\right), 1.50(9 \mathrm{H}, \mathrm{s}$, $\left.\mathrm{C}\left(\mathrm{CH}_{3}\right)_{3}\right), 1.63(1 \mathrm{H}, \mathrm{m}, \mathrm{C}-\mathrm{CHaHb}-\mathrm{C}), 2.15(1 \mathrm{H}, \mathrm{m}, \mathrm{C}-\mathrm{CHaHb}-\mathrm{C}), 2.47\left(1 \mathrm{H}, \mathrm{ddd},{ }^{2} J_{\mathrm{HH}}=13.2\right.$, ${ }^{3} J_{\mathrm{HH}}=9.6$ and $\left.6.8 \mathrm{~Hz}, \mathrm{~S}-\mathrm{C} \underline{\mathrm{HaHb}}\right), 2.54\left(1 \mathrm{H}\right.$, ddd, ${ }^{2} J_{\mathrm{HH}}=13.2,{ }^{3} J_{\mathrm{HH}}=10.0$ and $4.7 \mathrm{~Hz}, \mathrm{~S}-$ $\mathrm{C} \underline{\mathrm{HaHb}}), 3.71\left(2 \mathrm{H}, \mathrm{s}, \mathrm{S}-\mathrm{CH}_{2}\right), 3.90\left(1 \mathrm{H}, \mathrm{dtd},{ }^{3} J_{\mathrm{HP}}=16.8,{ }^{3} J_{\mathrm{HH}}=10.4,10.4\right.$ and $\left.3.6 \mathrm{~Hz}, \mathrm{~N}-\mathrm{CH}-\mathrm{P}\right)$, 
$4.54\left(1 \mathrm{H}, \mathrm{dd},{ }^{3} J_{\mathrm{HH}}=10.4,{ }^{3} J_{\mathrm{HP}}=3.3, \mathrm{NH}\right), 7.22\left(1 \mathrm{H}_{\text {arom }}, \mathrm{m}, 1 \mathrm{CH}\right), 7.30\left(4 \mathrm{H}_{\text {arom }}, \mathrm{m}, 4 \mathrm{CH}\right) .{ }^{13} \mathrm{C}$ NMR (150.9 MHz; $\left.\mathrm{CDCl}_{3}\right): \delta_{\mathrm{C}} 27.88\left(1 \mathrm{C}, \mathrm{d},{ }^{3} J_{\mathrm{CP}}=14.5, \mathrm{~S}_{-} \mathrm{CH}_{2}\right), 28.00\left(3 \mathrm{C}, \mathrm{C}\left(\underline{\mathrm{CH}}_{3}\right)_{3}\right), 30.15$ $\left(3 \mathrm{C}, \mathrm{d},{ }^{3} J_{\mathrm{CP}}=3.6, \mathrm{C}\left(\underline{\mathrm{CH}}_{3}\right)_{3}\right), 30.36\left(3 \mathrm{C}, \mathrm{d},{ }^{3} J_{\mathrm{CP}}=3.6, \mathrm{C}\left(\underline{\mathrm{CH}}_{3}\right)_{3}\right), 31.09\left(\mathrm{~d},{ }^{2} J_{\mathrm{CP}}=3.6 \mathrm{~Hz}, \mathrm{C}-\underline{C H}_{2}-\right.$ C), $36.17\left(1 \mathrm{C}, \mathrm{S}-\mathrm{CH}_{2}\right), 49.07\left(1 \mathrm{C}, \mathrm{d},{ }^{1} J_{\mathrm{CP}}=159.7 \mathrm{~Hz}, \mathrm{~N}-\mathrm{CH}-\mathrm{P}\right), 79.63\left(1 \mathrm{C}, \underline{\mathrm{C}}\left(\mathrm{CH}_{3}\right)_{3}\right), 82.47(1 \mathrm{C}$, $\left.\mathrm{d},{ }^{2} J_{\mathrm{CP}}=8.4 \mathrm{~Hz}, \underline{\mathrm{C}}\left(\mathrm{CH}_{3}\right)_{3}\right), 83.01\left(1 \mathrm{C}, \mathrm{d},{ }^{2} J_{\mathrm{CP}}=9.9 \mathrm{~Hz}, \underline{\mathrm{C}}\left(\mathrm{CH}_{3}\right)_{3}\right), 126.86\left(1 \mathrm{C}\right.$, para- $\left.\mathrm{CH}_{\text {arom }}\right)$, 128.40 (2C, ortho- $\mathrm{CH}_{\text {arom }}$ ), 128.81 (2C, meta- $\left.\mathrm{CH}_{\text {arom }}\right), 138.34$ (1C, ipso- $\left.\mathrm{C}_{\text {arom}}\right), 155.29$ (1C, d, $\left.{ }^{3} J_{\mathrm{CP}}=6.3 \mathrm{~Hz}, \mathrm{~N}-\mathrm{CO}-\mathrm{O}\right)$. IR $\left(\mathrm{CCl}_{4}, v_{\max }, \mathrm{cm}^{-1}\right) 3449 \mathrm{~m}, 3259 \mathrm{w}(\mathrm{NH}) ; 1530 \mathrm{~m}, 1496 \mathrm{vs}(\mathrm{CONH})$; 1718 vs $(\mathrm{C}=\mathrm{O}) ; 1259 \mathrm{~m}(\mathrm{P}=\mathrm{O}) ; 982$ vs $(\mathrm{CO}-\mathrm{P}) ; 1454 \mathrm{~s}, 1072 \mathrm{~m}, 918 \mathrm{~m}, 691 \mathrm{~s}$ (ring); $2981 \mathrm{~s}$, 1393 s, 1370 vs, $\left(\mathrm{CH}_{3}\right), 1171$ vs $\left(\mathrm{C}\left(\mathrm{CH}_{3}\right)_{3}\right)$. HRMS (ESI) calc for $\mathrm{C}_{23} \mathrm{H}_{40} \mathrm{O}_{5} \mathrm{NNaPS}[\mathrm{M}+\mathrm{Na}]^{+}$ 496.22570; found: 496.22551 .

Dimethyl $[(R, S)-1-($ benzyloxycarbonylamino)-3-(phenylsulfanyl)propyl]phosphonate (8a). Compound 8a was prepared using a slight modification of the method developed by Yuan et al. ${ }^{16}$ A well-stirred mixture of benzylcarbamate (3 g; $20 \mathrm{mmol})$ and dimethyl phosphite (2.2 $\mathrm{g} ; 20$ $\mathrm{mmol})$ in $50 \mathrm{ml}$ of acetyl chloride was cooled in ice bath to $-5{ }^{\circ} \mathrm{C}$, and aldehyde $1 \mathrm{a}(4.2 \mathrm{~g} ; 25$ mmol) was added dropwise over 10 minutes. The reaction mixture was stirred for 1 hour at $0^{\circ} \mathrm{C}$ and then left to react at rt overnight. The acetyl chloride was evaporated in vacuo, and the crude product was purified twice by flash chromatography on silica gel using a linear gradient of ethyl acetate in toluene as the eluant. The yield was $2.5 \mathrm{~g} \mathrm{(31 \% ).}$

Alternatively, phosphonate 8a was prepared as follows. Sodium methoxide (4 g; $74.8 \mathrm{mmol})$ was carefully added to a stirred solution of compound $4 \mathbf{a}(10 \mathrm{~g} ; 18.7 \mathrm{mmol})$ dissolved in $100 \mathrm{ml}$ of anhydrous methanol and $100 \mathrm{ml}$ of dry dioxane. The flask was equipped with a calcium dichloride tube and the reaction was allowed to proceed at $\mathrm{rt}$ overnight. The solvents were evaporated in vacuo; the residue was taken up in $100 \mathrm{ml}$ of diethyl ether and washed with $100 \mathrm{ml}$ of water and brine. The organic layer was separated, dried over $\mathrm{Na}_{2} \mathrm{SO}_{4}$, filtered and evaporated in vacuo. The crude product was purified by flash chromatography on silica gel using a linear gradient of ethyl acetate in toluene as the eluant. Yield $5.5 \mathrm{~g}(71 \%)$. Colorless oil, $\mathrm{R}_{\mathrm{f}}=0.59$ (S3). ${ }^{1} \mathrm{H}$ NMR (600 MHz; $\mathrm{CDCl}_{3}$ ): $\delta_{\mathrm{H}} 1.89$ (1H, m, C-C $\left.\underline{\mathrm{HaHb}}-\mathrm{C}\right), 2.13$ (1H, m, C-CHaHb-C), 2.93 $\left(1 \mathrm{H}, \mathrm{ddd},{ }^{2} J_{\mathrm{HH}}=13.4,{ }^{3} J_{\mathrm{HH}}=9.0\right.$ and $\left.7.0 \mathrm{~Hz}, \mathrm{~S}-\mathrm{CHaHb}\right), 3.06\left(1 \mathrm{H}, \mathrm{ddd},{ }^{2} J_{\mathrm{HH}}=13.4,{ }^{3} J_{\mathrm{HH}}=9.1\right.$ and $4.8 \mathrm{~Hz}, \mathrm{~S}-\mathrm{CHaHb}), 3.69\left(3 \mathrm{H}, \mathrm{d},{ }^{3} J_{\mathrm{HP}}=10.7, \mathrm{OCH}_{3}\right), 3.71\left(3 \mathrm{H}, \mathrm{d},{ }^{3} J_{\mathrm{HP}}=10.7, \mathrm{OCH}_{3}\right), 4.30$ $\left(1 \mathrm{H}, \mathrm{dtd},{ }^{3} J_{\mathrm{HP}}=16.8,{ }^{3} J_{\mathrm{HH}}=10.4,10.4\right.$ and $\left.3.9 \mathrm{~Hz}, \mathrm{~N}-\mathrm{CH}-\mathrm{P}\right), 5.11\left(1 \mathrm{H}, \mathrm{d},{ }^{2} J_{\mathrm{HH}}=12.4\right.$, O$\mathrm{C} \underline{\mathrm{HaHb}}), 5.14\left(1 \mathrm{H}, \mathrm{d},{ }^{2} J_{\mathrm{HH}}=12.4, \mathrm{O}-\mathrm{CHaHb}\right), 5.26\left(1 \mathrm{H}, \mathrm{dd},{ }^{3} J_{\mathrm{HH}}=10.4,{ }^{3} J_{\mathrm{HP}}=1.9, \mathrm{NH}\right), 7.19$ $\left(1 \mathrm{H}_{\text {arom }}, \mathrm{m}, 1 \mathrm{CH}\right), 7.27\left(2 \mathrm{H}_{\text {arom }}, \mathrm{m}, 2 \mathrm{CH}\right), 7.32\left(1 \mathrm{H}_{\text {arom }}, \mathrm{m}, 1 \mathrm{CH}\right), 7.33\left(2 \mathrm{H}_{\text {arom }}, \mathrm{m}, 2 \mathrm{CH}\right), 7.35$ $\left(4 \mathrm{H}_{\text {arom }}, \mathrm{m}, 4 \mathrm{CH}\right) .{ }^{13} \mathrm{C} \mathrm{NMR}\left(150.9 \mathrm{MHz} ; \mathrm{CDCl}_{3}\right): \delta_{\mathrm{C}} 29.74\left(1 \mathrm{C}, \mathrm{d},{ }^{2} J_{\mathrm{CP}}=4.2 \mathrm{~Hz}, \mathrm{C}-\underline{\mathrm{CH}}_{2}-\mathrm{C}\right)$, $30.21\left(1 \mathrm{C}, \mathrm{d},{ }^{3} J_{\mathrm{CP}}=14.3, \mathrm{~S}-\mathrm{CH}_{2}\right), 46.34\left(1 \mathrm{C}, \mathrm{d},{ }^{1} J_{\mathrm{CP}}=156.7 \mathrm{~Hz}, \mathrm{~N}-\mathrm{CH}-\mathrm{P}\right), 79.63\left(1 \mathrm{C}, \underline{\mathrm{C}}\left(\mathrm{CH}_{3}\right)_{3}\right)$, $53.07\left(1 \mathrm{C}, \mathrm{d},{ }^{2} J_{\mathrm{CP}}=6.6 \mathrm{~Hz}, \mathrm{OCH}_{3}\right), 53.25\left(1 \mathrm{C}, \mathrm{d},{ }^{2} J_{\mathrm{CP}}=7.1 \mathrm{~Hz}, \mathrm{OCH}_{3}\right), 67.26\left(1 \mathrm{C}, \mathrm{OCH}_{2}\right)$, $126.32,128.05(2), 128.23,128.50(2), 128.94(2)$ and 129.75(2) $\left(10 \mathrm{C}, \mathrm{CH}_{\text {arom }}\right), 135.38$ (1C, ipso$\left.\mathrm{C}_{\text {arom}}\right), 136.07$ (1C, ipso- $\left.\mathrm{C}_{\text {arom }}\right), 155.97\left(1 \mathrm{C}, \mathrm{d},{ }^{3} J_{\mathrm{CP}}=5.5 \mathrm{~Hz}, \mathrm{~N}-\mathrm{CO}-\mathrm{O}\right)$. IR $\left(\mathrm{CCl}_{4}, v_{\max }, \mathrm{cm}^{-1}\right)$ 3433 w, $3240 \mathrm{~m}(\mathrm{NH}) ; 1540 \mathrm{~m}, 1504 \mathrm{~m}(\mathrm{CONH}) ; 1724 \mathrm{vs}(\mathrm{C}=\mathrm{O}) ; 1252 \mathrm{~s}, 1233 \mathrm{~s}(\mathrm{P}=\mathrm{O}) ; 1044 \mathrm{vs}$ (C-OP); 835 m (CO-P); 1481 m, 1455 m, 1440 m, 695 s (ring); 2955 m ( $\left.\mathrm{CH}_{3}\right)$. HRMS (ESI) calc for $\mathrm{C}_{19} \mathrm{H}_{25} \mathrm{O}_{5} \mathrm{NPS}[\mathrm{M}+1]^{+} 410.11856$; found: 410.11850 . 
Dimethyl [(R,S)-1-(benzyloxycarbonylamino)-3-(tert-butylsulfanyl)propyl]phosphonate (8b). Phosphonate $\mathbf{8 b}$ was prepared in the same manner as $\mathbf{8 a}$ by the reaction of benzylcarbamate (3 g; $20 \mathrm{mmol})$ and dimethyl phosphite $(2.2 \mathrm{~g} ; 20 \mathrm{mmol})$ and aldehyde $1 \mathbf{b}$ (3.7 g; $25 \mathrm{mmol})$. The yield was $3.5 \mathrm{~g}(45 \%)$.

Phosphonate $\mathbf{8 b}$ was also prepared using the alternative protocol for $\mathbf{8 a}$ by the reaction of sodium methoxide (2.7 g; $51.6 \mathrm{mmol})$ and phosphonate 4b (6.6 g; $12.9 \mathrm{mmol})$. Yield $3 \mathrm{~g}(66 \%)$. Colorless oil, $\mathrm{R}_{\mathrm{f}}=0.28(\mathrm{~S} 2) .{ }^{1} \mathrm{H}$ NMR $\left(600 \mathrm{MHz} ; \mathrm{CDCl}_{3}\right): \delta_{\mathrm{H}} 1.29\left(9 \mathrm{H}, \mathrm{s}, \mathrm{C}\left(\mathrm{CH}_{3}\right)_{3}\right), 1.86(1 \mathrm{H}$, $\mathrm{m}, \mathrm{C}-\mathrm{CHaHb}-\mathrm{C}), 2.08(1 \mathrm{H}, \mathrm{m}, \mathrm{C}-\mathrm{CHaHb}-\mathrm{C}), 2.56\left(1 \mathrm{H}, \mathrm{ddd},{ }^{2} J_{\mathrm{HH}}=12.1,{ }^{3} J_{\mathrm{HH}}=9.0\right.$ and $7.2 \mathrm{~Hz}$, $\mathrm{S}-\mathrm{C} \underline{\mathrm{HaHb}}), 2.66\left(1 \mathrm{H}, \mathrm{ddd},{ }^{2} J_{\mathrm{HH}}=12.1,{ }^{3} J_{\mathrm{HH}}=9.2\right.$ and $\left.5.0 \mathrm{~Hz}, \mathrm{~S}-\mathrm{CHaHb}\right), 3.72\left(3 \mathrm{H}, \mathrm{d},{ }^{3} J_{\mathrm{HP}}=\right.$ 10.6, $\left.\mathrm{OCH}_{3}\right), 3.75\left(3 \mathrm{H}, \mathrm{d},{ }^{3} J_{\mathrm{HP}}=10.6, \mathrm{OCH}_{3}\right), 4.26\left(1 \mathrm{H}, \mathrm{dtd},{ }^{3} J_{\mathrm{HP}}=16.5,{ }^{3} J_{\mathrm{HH}}=10.4,10.4\right.$ and $3.8 \mathrm{~Hz}, \mathrm{~N}-\mathrm{CH}-\mathrm{P}), 5.13\left(2 \mathrm{H}, \mathrm{s}, \mathrm{O}-\mathrm{CH}_{2}\right), 5.25\left(1 \mathrm{H}, \mathrm{dd},{ }^{3} J_{\mathrm{HH}}=10.4,{ }^{3} J_{\mathrm{HP}}=1.5, \mathrm{NH}\right), 7.30-7.36$ $\left(5 \mathrm{H}_{\text {arom }}, \mathrm{m}, 5 \mathrm{CH}\right) .{ }^{13} \mathrm{C} \mathrm{NMR}\left(150.9 \mathrm{MHz} ; \mathrm{CDCl}_{3}\right): \delta_{\mathrm{C}} 24.58\left(1 \mathrm{C}, \mathrm{d},{ }^{3} J_{\mathrm{CP}}=14.3, \mathrm{~S}-\mathrm{CH}_{2}\right), 30.41$ $\left(1 \mathrm{C}, \mathrm{d},{ }^{2} J_{\mathrm{CP}}=4.0 \mathrm{~Hz}, \mathrm{C}-\underline{C H}_{2}-\mathrm{C}\right), 30.87\left(3 \mathrm{C}, \mathrm{C}\left(\underline{\mathrm{CH}}_{3}\right)_{3}\right), 42.32\left(1 \mathrm{C}, \underline{\mathrm{C}}\left(\mathrm{CH}_{3}\right)_{3}\right), 46.71\left(1 \mathrm{C}, \mathrm{d},{ }^{1} J_{\mathrm{CP}}=\right.$ $156.4 \mathrm{~Hz}, \mathrm{~N}-\mathrm{CH}-\mathrm{P}), 53.05\left(1 \mathrm{C}, \mathrm{d},{ }^{2} J_{\mathrm{CP}}=6.6 \mathrm{~Hz}, \mathrm{OCH}_{3}\right), 53.22\left(1 \mathrm{C}, \mathrm{d},{ }^{2} J_{\mathrm{CP}}=7.1 \mathrm{~Hz}, \mathrm{OCH}_{3}\right)$, $67.15\left(1 \mathrm{C}, \mathrm{OCH}_{2}\right), 128.01\left(2 \mathrm{C}\right.$, meta- $\left.\mathrm{CH}_{\text {arom }}\right), 128.16\left(1 \mathrm{C}\right.$, para $\left.-\mathrm{CH}_{\text {arom }}\right), 128.46(2 \mathrm{C}$, ortho$\left.\mathrm{CH}_{\text {arom }}\right), 136.16$ (1C, ipso-C $\left.\mathrm{C}_{\text {arom }}\right), 155.93\left(1 \mathrm{C}, \mathrm{d},{ }^{3} J_{\mathrm{CP}}=5.4 \mathrm{~Hz}, \mathrm{~N}-\mathrm{CO}-\mathrm{O}\right) . \mathrm{IR}\left(\mathrm{CCl}_{4}, v_{\max }, \mathrm{cm}^{-1}\right)$ $3433 \mathrm{w}, 3240 \mathrm{~m}(\mathrm{NH}) ; 1540 \mathrm{~m}, 1505 \mathrm{~s}(\mathrm{CONH}) ; 1725$ vs $(\mathrm{C}=\mathrm{O}) ; 1252 \mathrm{~s}(\mathrm{P}=\mathrm{O}) ; 1044$ vs $(\mathrm{C}-\mathrm{OP})$; $835 \mathrm{~m}$ (CO-P); $1458 \mathrm{~m}, 696 \mathrm{~m}$ (ring); $2957 \mathrm{~s}\left(\mathrm{CH}_{3}\right) ; 1165 \mathrm{~m}\left(\mathrm{C}\left(\mathrm{CH}_{3}\right)_{3}\right)$. HRMS (ESI) calc for $\mathrm{C}_{17} \mathrm{H}_{29} \mathrm{O}_{5} \mathrm{NPS}[\mathrm{M}+1]^{+}$390.14986; found: 390.14986.

Dimethyl [(R,S)-1-(benzyloxycarbonylamino)-3-(benzylsulfanyl)propyl]phosphonate (8c). Phosphonate 8c was prepared in the same manner as $8 \mathbf{a}$ by the reaction of benzylcarbamate (4 g; $26.5 \mathrm{mmol}$ ) and dimethyl phosphite $(2.9 \mathrm{~g} ; 26.5 \mathrm{mmol})$ and aldehyde $1 \mathrm{c}(5.7 \mathrm{~g} ; 31.8 \mathrm{mmol})$. The yield was $4.5 \mathrm{~g}(40 \%)$.

Phosphonate 8c was also prepared using the alternative protocol for 8a by the reaction of sodium methoxide (4 g; $73 \mathrm{mmol})$ and phosphonate 4c (10 g; $18.2 \mathrm{mmol})$. Yield $5 \mathrm{~g}$ (65\%). Colorless oil, $\mathrm{R}_{\mathrm{f}}=0.65(\mathrm{~S} 3) .{ }^{1} \mathrm{H}$ NMR $\left(600 \mathrm{MHz} ; \mathrm{CDCl}_{3}\right): \delta_{\mathrm{H}} 1.81(1 \mathrm{H}, \mathrm{m}, \mathrm{C}-\mathrm{CHaHb}-\mathrm{C}), 2.05$ (1H, m, CCHa $\underline{\mathrm{Hb}}-\mathrm{C}), 2.44\left(1 \mathrm{H}, \mathrm{ddd},{ }^{2} J_{\mathrm{HH}}=13.4,{ }^{3} J_{\mathrm{HH}}=8.8\right.$ and $\left.7.3 \mathrm{~Hz}, \mathrm{~S}-\mathrm{CHaHb}\right), 2.54\left(1 \mathrm{H}, \mathrm{ddd},{ }^{2} J_{\mathrm{HH}}=\right.$ $13.4,{ }^{3} J_{\mathrm{HH}}=9.0$ and $\left.4.8 \mathrm{~Hz}, \mathrm{~S}-\mathrm{CHaHb}\right), 3.38\left(3 \mathrm{H}, \mathrm{d},{ }^{3} J_{\mathrm{HP}}=10.8, \mathrm{OCH}_{3}\right), 3.69\left(2 \mathrm{H}, \mathrm{s}, \mathrm{S}-\mathrm{CH}_{2}\right)$, $3.72\left(3 \mathrm{H}, \mathrm{d},{ }^{3} J_{\mathrm{HP}}=10.8, \mathrm{OCH}_{3}\right), 4.23\left(1 \mathrm{H}, \mathrm{dtd},{ }^{3} J_{\mathrm{HP}}=16.5,{ }^{3} J_{\mathrm{HH}}=10.4,10.4\right.$ and $3.7 \mathrm{~Hz}, \mathrm{~N}-\mathrm{CH}-$ P), $5.09\left(1 \mathrm{H}, \mathrm{d},{ }^{2} J_{\mathrm{HH}}=12.2, \mathrm{O}-\mathrm{CHaHb}\right), 5.12\left(1 \mathrm{H}, \mathrm{d},{ }^{2} J_{\mathrm{HH}}=12.2, \mathrm{O}-\mathrm{CHaHb}\right), 5.35\left(1 \mathrm{H}, \mathrm{dd},{ }^{3} J_{\mathrm{HH}}\right.$ $\left.=10.4,{ }^{3} J_{\mathrm{HP}}=1.2, \mathrm{NH}\right), 7.22-7.34\left(10 \mathrm{H}_{\text {arom }}, \mathrm{m}, 10 \mathrm{CH}\right) .{ }^{13} \mathrm{C} \mathrm{NMR}\left(150.9 \mathrm{MHz} ; \mathrm{CDCl}_{3}\right): \delta_{\mathrm{C}}$ $27.30\left(1 \mathrm{C}, \mathrm{d},{ }^{3} J_{\mathrm{CP}}=14.2, \mathrm{~S}-\mathrm{CH}_{2}\right), 29.60\left(1 \mathrm{C}, \mathrm{d},{ }^{2} J_{\mathrm{CP}}=4.0 \mathrm{~Hz}, \mathrm{C}-\underline{C H}_{2}-\mathrm{C}\right), 35.98\left(1 \mathrm{C}, \mathrm{S}-\mathrm{CH}_{2}\right)$, $46.25\left(1 \mathrm{C}, \mathrm{d},{ }^{1} J_{\mathrm{CP}}=156.8 \mathrm{~Hz}, \mathrm{~N}-\mathrm{CH}-\mathrm{P}\right), 52.96\left(1 \mathrm{C}, \mathrm{d},{ }^{2} J_{\mathrm{CP}}=6.6 \mathrm{~Hz}, \mathrm{OCH}_{3}\right), 53.19\left(1 \mathrm{C}, \mathrm{d},{ }^{2} J_{\mathrm{CP}}=\right.$ $\left.7.1 \mathrm{~Hz}, \mathrm{OCH}_{3}\right), 67.10\left(1 \mathrm{C}, \mathrm{OCH}_{2}\right), 129.91,127.98(2), 128.14,128.40(2), 128.42(2)$ and 128.77 (2) $\left(10 \mathrm{C}, \mathrm{CH}_{\text {arom }}\right), 136.09$ (1C, ipso- $\left.\mathrm{C}_{\text {arom }}\right), 138.02$ (1C, ipso- $\left.\mathrm{C}_{\text {arom }}\right), 155.90\left(1 \mathrm{C}, \mathrm{d},{ }^{3} J_{\mathrm{CP}}=5.3 \mathrm{~Hz}\right.$, N-CO-O). IR ( $\left.\mathrm{CCl}_{4}, v_{\max }, \mathrm{cm}^{-1}\right) 3434 \mathrm{w}, 3242 \mathrm{~m}(\mathrm{NH}) ; 1540 \mathrm{~m}, 1497 \mathrm{~s}(\mathrm{CONH}) ; 1724 \mathrm{vs}(\mathrm{C}=\mathrm{O})$; $1252 \mathrm{~s}(\mathrm{P}=\mathrm{O}) ; 1043$ vs (C-OP); $834 \mathrm{~m}(\mathrm{CO}-\mathrm{P}) ; 1454 \mathrm{~m}, 698 \mathrm{~s}$ (ring). HRMS (ESI) calc for $\mathrm{C}_{20} \mathrm{H}_{27} \mathrm{O}_{5} \mathrm{NPS}[\mathrm{M}+1]^{+} 424.13421$; found: 424.13409 .

Dimethyl [(R,S)-1-(tert-butoxycarbonylamino)-3-(phenylsulfanyl)propyl]phosphonate (9a). Sodium methoxide (4.3 g; $80 \mathrm{mmol}$ ) was carefully added to a stirred solution of compound $6 \mathbf{6}$ 
(10 g; $20 \mathrm{mmol}$ ) dissolved in $100 \mathrm{ml}$ of anhydrous methanol and $100 \mathrm{ml}$ of dry dioxane. The flask was equipped with a calcium dichloride tube and the reaction was allowed to proceed at $\mathrm{rt}$ overnight. The solvents were evaporated in vacuo; the residue was taken up in $100 \mathrm{ml}$ of diethyl ether and washed with $100 \mathrm{ml}$ of water and brine. The organic layer was separated, dried over $\mathrm{Na}_{2} \mathrm{SO}_{4}$, filtered and evaporated in vacuo. The crude product was purified by flash chromatography on silica gel using a linear gradient of ethyl acetate in toluene as the eluant. Yield $4.1 \mathrm{~g}(55 \%)$. Colorless oil, $\mathrm{R}_{\mathrm{f}}=0.65(\mathrm{~S} 3) .{ }^{1} \mathrm{H}$ NMR $\left(600 \mathrm{MHz} ; \mathrm{CDCl}_{3}\right): \delta_{\mathrm{H}} 1.45(9 \mathrm{H}, \mathrm{s}$, $\left.\mathrm{C}\left(\mathrm{CH}_{3}\right)_{3}\right), 1.86(1 \mathrm{H}, \mathrm{m}, \mathrm{C}-\mathrm{CHaHb}-\mathrm{C}), 2.13(1 \mathrm{H}, \mathrm{m}, \mathrm{C}-\mathrm{CHaHb}-\mathrm{C}), 2.95\left(1 \mathrm{H}, \mathrm{ddd},{ }^{2} J_{\mathrm{HH}}=13.4\right.$, ${ }^{3} J_{\mathrm{HH}}=9.2$ and $\left.6.9 \mathrm{~Hz}, \mathrm{SC} \underline{\mathrm{HaHb}}\right), 3.07\left(1 \mathrm{H}, \mathrm{ddd},{ }^{2} J_{\mathrm{HH}}=13.4,{ }^{3} J_{\mathrm{HH}}=9.4\right.$ and $\left.4.8 \mathrm{~Hz}, \mathrm{~S}-\mathrm{C} \underline{\mathrm{HaHb}}\right)$, $3.74\left(3 \mathrm{H}, \mathrm{d},{ }^{3} J_{\mathrm{HP}}=10.6, \mathrm{OCH}_{3}\right), 3.76\left(3 \mathrm{H}, \mathrm{d},{ }^{3} J_{\mathrm{HP}}=10.6, \mathrm{OCH}_{3}\right), 4.24\left(1 \mathrm{H}, \mathrm{dtd},{ }^{3} J_{\mathrm{HP}}=17.0\right.$, ${ }^{3} J_{\mathrm{HH}}=10.5,10.4$ and $\left.4.0 \mathrm{~Hz}, \mathrm{~N}-\mathrm{CH}-\mathrm{P}\right), 4.84\left(1 \mathrm{H}, \mathrm{dd},{ }^{3} J_{\mathrm{HH}}=10.5,{ }^{3} J_{\mathrm{HP}}=1.7, \mathrm{NH}\right), 7.19\left(1 \mathrm{H}_{\text {arom, }}\right.$ $\mathrm{m}, 1 \mathrm{CH}), 7.28\left(2 \mathrm{H}_{\text {arom }}, \mathrm{m}, 2 \mathrm{CH}\right), 7.35\left(2 \mathrm{H}_{\text {arom }}, \mathrm{m}, 2 \mathrm{CH}\right) .{ }^{13} \mathrm{C} \mathrm{NMR}\left(150.9 \mathrm{MHz} ; \mathrm{CDCl}_{3}\right): \delta_{\mathrm{C}}$ $28.20\left(3 \mathrm{C}, \mathrm{C}\left(\underline{\mathrm{CH}}_{3}\right)_{3}\right), 29.96\left(1 \mathrm{C}, \mathrm{d},{ }^{2} J_{\mathrm{CP}}=4.4 \mathrm{~Hz}, \mathrm{C}-\underline{C H}_{2}-\mathrm{C}\right), 30.24\left(1 \mathrm{C}, \mathrm{d},{ }^{3} J_{\mathrm{CP}}=14.4, \mathrm{~S}-\mathrm{CH}_{2}\right)$, $45.27\left(1 \mathrm{C}, \mathrm{d},{ }^{1} J_{\mathrm{CP}}=156.0 \mathrm{~Hz}, \mathrm{~N}-\mathrm{CH}-\mathrm{P}\right), 52.97\left(1 \mathrm{C}, \mathrm{d},{ }^{2} J_{\mathrm{CP}}=6.6 \mathrm{~Hz}, \mathrm{OCH}_{3}\right), 53.24\left(1 \mathrm{C}, \mathrm{d},{ }^{2} J_{\mathrm{CP}}=\right.$ $\left.7.0 \mathrm{~Hz}, \mathrm{OCH}_{3}\right), 80.30\left(1 \mathrm{C}, \underline{\mathrm{C}}\left(\mathrm{CH}_{3}\right)_{3}\right), 126.26\left(1 \mathrm{C}\right.$, para- $\left.\mathrm{CH}_{\text {arom }}\right), 128.91$ (2C, meta- $\left.\mathrm{CH}_{\text {arom }}\right)$, 129.71 (2C, ortho- $\left.\mathrm{CH}_{\text {arom }}\right), 135.52$ (1C, ipso- $\left.\mathrm{C}_{\text {arom }}\right), 155.24\left(1 \mathrm{C}, \mathrm{d},{ }^{3} J_{\mathrm{CP}}=5.7 \mathrm{~Hz}, \mathrm{~N}-\mathrm{CO}-\mathrm{O}\right)$. IR $\left(\mathrm{CCl}_{4}, v_{\max }, \mathrm{cm}^{-1}\right) 3436 \mathrm{w}, 3258 \mathrm{~m}(\mathrm{NH}) ; 1529 \mathrm{~m}, 1496 \mathrm{~s}(\mathrm{CONH}) ; 1718 \mathrm{vs}(\mathrm{C}=\mathrm{O}) ; 1251 \mathrm{~s}$ $(\mathrm{P}=\mathrm{O}) ; 1062$ vs, 1045 vs, 1037 vs (C-OP); $834 \mathrm{~m}$ (CO-P); $1482 \mathrm{~s}, 1440 \mathrm{~m}, 691 \mathrm{~m}$ (ring); $2980 \mathrm{~m}$,

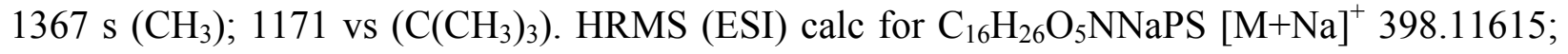
found: 398.11617.

Dimethyl [(R,S)-1-(tert-butoxycarbonylamino)-3-(tert-butylsulfanyl)propyl]phosphonate (9b). Phosphonate $9 \mathbf{b}$ was prepared in the same manner as $9 \mathbf{a}$ by the reaction of compound $\mathbf{6 b}$ (7.3 g; $15.2 \mathrm{mmol})$ and sodium methoxide (3.3 g; $60.8 \mathrm{mmol})$. Yield $3.5 \mathrm{~g} \mathrm{(64 \% ).} \mathrm{Colorless} \mathrm{oil,}$ $\mathrm{R}_{\mathrm{f}}=0.67(\mathrm{~S} 3) .{ }^{1} \mathrm{H} \mathrm{NMR}\left(600 \mathrm{MHz} ; \mathrm{CDCl}_{3}\right): \delta_{\mathrm{H}} 1.31\left(9 \mathrm{H}, \mathrm{s}, \mathrm{C}\left(\mathrm{CH}_{3}\right)_{3}\right), 1.45\left(9 \mathrm{H}, \mathrm{s}, \mathrm{C}\left(\mathrm{CH}_{3}\right)_{3}\right)$, $1.82(1 \mathrm{H}, \mathrm{m}, \mathrm{C}-\mathrm{C} \underline{\mathrm{HaHb}}-\mathrm{C}), 2.08(1 \mathrm{H}, \mathrm{m}, \mathrm{C}-\mathrm{CHaHb}-\mathrm{C}), 2.58\left(1 \mathrm{H}, \mathrm{ddd},{ }^{2} J_{\mathrm{HH}}=12.2,{ }^{3} J_{\mathrm{HH}}=9.2\right.$ and $7.3 \mathrm{~Hz}, \mathrm{~S}-\mathrm{C} \underline{\mathrm{HaHb}}), 2.68\left(1 \mathrm{H}, \mathrm{ddd},{ }^{2} \mathrm{~J}_{\mathrm{HH}}=12.2,{ }^{3} \mathrm{~J}_{\mathrm{HH}}=9.3\right.$ and $\left.4.9 \mathrm{~Hz}, \mathrm{~S}-\mathrm{CHaHb}\right), 3.73(6 \mathrm{H}$, $\left.\mathrm{d},{ }^{3} J_{\mathrm{HP}}=10.5,2 \mathrm{x} \mathrm{OCH}_{3}\right), 4.21\left(1 \mathrm{H}, \mathrm{dtd},{ }^{3} J_{\mathrm{HP}}=16.6,{ }^{3} J_{\mathrm{HH}}=10.5,10.5\right.$ and $\left.3.8 \mathrm{~Hz}, \mathrm{~N}-\mathrm{CH}-\mathrm{P}\right)$, $4.76\left(1 \mathrm{H}, \mathrm{bd},{ }^{3} J_{\mathrm{HH}}=10.5, \mathrm{NH}\right) .{ }^{13} \mathrm{C} \mathrm{NMR}\left(150.9 \mathrm{MHz} ; \mathrm{CDCl}_{3}\right): \delta_{\mathrm{C}} 24.62\left(1 \mathrm{C}, \mathrm{d},{ }^{3} J_{\mathrm{CP}}=14.3\right.$, S$\left.\mathrm{CH}_{2}\right), 28.22\left(3 \mathrm{C}, \mathrm{C}\left(\underline{\mathrm{CH}}_{3}\right)_{3}\right), 30.67\left(1 \mathrm{C}, \mathrm{d},{ }^{2} J_{\mathrm{CP}}=4.2 \mathrm{~Hz}, \mathrm{C}-\underline{C H}_{2}-\mathrm{C}\right), 30.92\left(3 \mathrm{C}, \mathrm{C}\left(\underline{\mathrm{CH}}_{3}\right)_{3}\right), 42.29$ $\left(1 \mathrm{C}, \underline{\mathrm{C}}\left(\mathrm{CH}_{3}\right)_{3}\right), 46.10\left(1 \mathrm{C}, \mathrm{d},{ }^{1} J_{\mathrm{CP}}=155.8 \mathrm{~Hz}, \mathrm{~N}-\mathrm{CH}-\mathrm{P}\right), 52.98\left(1 \mathrm{C}, \mathrm{d},{ }^{2} J_{\mathrm{CP}}=6.6 \mathrm{~Hz}, \mathrm{OCH}_{3}\right)$, $53.24\left(1 \mathrm{C}, \mathrm{d},{ }^{2} J_{\mathrm{CP}}=7.0 \mathrm{~Hz}, \mathrm{OCH}_{3}\right), 80.20\left(1 \mathrm{C}, \underline{\mathrm{C}}\left(\mathrm{CH}_{3}\right)_{3}\right), 155.24\left(1 \mathrm{C}, \mathrm{d},{ }^{3} J_{\mathrm{CP}}=5.4 \mathrm{~Hz}, \mathrm{~N}-\mathrm{CO}-\right.$ O). IR ( $\left.\mathrm{CCl}_{4}, v_{\max }, \mathrm{cm}^{-1}\right) 3437 \mathrm{~m}, 3259 \mathrm{~m}(\mathrm{NH}) ; 1529 \mathrm{~m}, 1497 \mathrm{~s}(\mathrm{CONH}) ; 1716 \mathrm{vs}(\mathrm{C}=\mathrm{O}) ; 1251$ vs $(\mathrm{P}=\mathrm{O}) ; 1062$ vs, 1046 vs $(\mathrm{C}-\mathrm{OP}) ; 834$ s $(\mathrm{CO}-\mathrm{P}) ; 2976$ s, 1366 vs $\left(\mathrm{CH}_{3}\right) ; 1172$ vs $\left(\mathrm{C}\left(\mathrm{CH}_{3}\right)_{3}\right)$. HRMS (ESI) calc for $\mathrm{C}_{14} \mathrm{H}_{30} \mathrm{O}_{5} \mathrm{NNaPS}[\mathrm{M}+\mathrm{Na}]^{+} 378.14745$; found: 378.14736 .

Dimethyl [(R,S)-1-( tert-butoxycarbonylamino)-3-(benzylsulfanyl)propyl]phosphonate (9c). Phosphonate 9c was prepared in the same manner as 9a by the reaction of compound $\mathbf{6 c}$ (7.3 $\mathrm{g}$; $14.2 \mathrm{mmol}$ ) and sodium methoxide $(3 \mathrm{~g} ; 56.8 \mathrm{mmol})$. Yield $3.1 \mathrm{~g}(57 \%)$. Colorless oil, $\mathrm{R}_{\mathrm{f}}=0.83$ (S3). ${ }^{1} \mathrm{H}$ NMR (600 MHz; $\left.\mathrm{CDCl}_{3}\right): \delta_{\mathrm{H}} 1.43\left(9 \mathrm{H}, \mathrm{s}, \mathrm{C}\left(\mathrm{CH}_{3}\right)_{3}\right), 1.77(1 \mathrm{H}, \mathrm{m}, \mathrm{C}-\mathrm{CHaHb}-\mathrm{C}), 2.06$ $(1 \mathrm{H}, \mathrm{m}, \mathrm{C}-\mathrm{CHaHb}-\mathrm{C}), 2.46\left(1 \mathrm{H}, \mathrm{ddd},{ }^{2} J_{\mathrm{HH}}=13.3,{ }^{3} J_{\mathrm{HH}}=9.0\right.$ and $\left.7.2 \mathrm{~Hz}, \mathrm{~S}-\mathrm{C} \underline{\mathrm{HaHb}}\right), 2.55(1 \mathrm{H}$, $\mathrm{ddd},{ }^{2} J_{\mathrm{HH}}=13.3,{ }^{3} J_{\mathrm{HH}}=9.3$ and $\left.4.8 \mathrm{~Hz}, \mathrm{~S}-\mathrm{CHaHb}\right), 3.75\left(3 \mathrm{H}, \mathrm{d},{ }^{3} J_{\mathrm{HP}}=10.6, \mathrm{OCH}_{3}\right), 3.76(3 \mathrm{H}$, 
$\left.\mathrm{d},{ }^{3} J_{\mathrm{HP}}=10.6, \mathrm{OCH}_{3}\right), 4.17\left(1 \mathrm{H}, \mathrm{dtd},{ }^{3} J_{\mathrm{HP}}=16.7,{ }^{3} J_{\mathrm{HH}}=10.5,10.5\right.$ and $\left.3.8 \mathrm{~Hz}, \mathrm{~N}-\mathrm{CH}-\mathrm{P}\right), 4.71$ $\left(1 \mathrm{H}, \mathrm{bd},{ }^{3} J_{\mathrm{HH}}=10.5, \mathrm{NH}\right), 7.23\left(1 \mathrm{H}_{\text {arom }}, \mathrm{m}, 1 \mathrm{CH}\right), 7.30\left(4 \mathrm{H}_{\text {arom }}, \mathrm{m}, 4 \mathrm{CH}\right) .{ }^{13} \mathrm{C} \mathrm{NMR}(150.9$ $\left.\mathrm{MHz} ; \mathrm{CDCl}_{3}\right): \delta_{\mathrm{C}} 27.44\left(1 \mathrm{C}, \mathrm{d},{ }^{3} J_{\mathrm{CP}}=14.5, \mathrm{~S}-\mathrm{CH}_{2}\right), 28.22\left(3 \mathrm{C}, \mathrm{C}\left(\underline{\mathrm{CH}}_{3}\right)_{3}\right), 30.04\left(1 \mathrm{C}, \mathrm{d},{ }^{2} J_{\mathrm{CP}}=\right.$ $\left.4.1 \mathrm{~Hz}, \mathrm{C}-\underline{\mathrm{CH}}_{2}-\mathrm{C}\right), 36.14\left(1 \mathrm{C}, \mathrm{S}-\mathrm{CH}_{2}\right), 45.76\left(1 \mathrm{C}, \mathrm{d},{ }^{1} J_{\mathrm{CP}}=156.0 \mathrm{~Hz}, \mathrm{~N}-\mathrm{CH}-\mathrm{P}\right), 52.95$ (1C, d, $\left.{ }^{2} J_{\mathrm{CP}}=6.8 \mathrm{~Hz}, \mathrm{OCH}_{3}\right), 53.23\left(1 \mathrm{C}, \mathrm{d},{ }^{2} J_{\mathrm{CP}}=7.0 \mathrm{~Hz}, \mathrm{OCH}_{3}\right), 80.22\left(1 \mathrm{C}, \underline{\mathrm{C}}\left(\mathrm{CH}_{3}\right)_{3}\right), 126.98(1 \mathrm{C}$, para- $\left.\mathrm{CH}_{\text {arom }}\right), 128.48$ (2C, ortho- $\left.\mathrm{CH}_{\text {arom }}\right), 128.84$ (2C, meta- $\left.\mathrm{CH}_{\text {arom }}\right), 138.16$ (1C, ipso- $\left.\mathrm{C}_{\text {arom}}\right)$, $155.20\left(1 \mathrm{C}, \mathrm{d},{ }^{3} J_{\mathrm{CP}}=5.3 \mathrm{~Hz}, \mathrm{~N}-\mathrm{CO}-\mathrm{O}\right)$. IR $\left(\mathrm{CCl}_{4}, v_{\max }, \mathrm{cm}^{-1}\right) 3437 \mathrm{w}, 3260 \mathrm{~m}(\mathrm{NH}) ; 1529 \mathrm{~m}$, 1496 s $(\mathrm{CONH}) ; 1716$ vs $(\mathrm{C}=\mathrm{O}) ; 1250$ vs $(\mathrm{P}=\mathrm{O}) ; 1062$ vs, 1045 vs (C-OP); 834 s (CO-P); 1454 s, $1440 \mathrm{~m}, 699 \mathrm{~s}$ (ring); $2980 \mathrm{~s}, 1367 \mathrm{~s}\left(\mathrm{CH}_{3}\right) ; 1173$ vs $\left(\mathrm{C}\left(\mathrm{CH}_{3}\right)_{3}\right)$. HRMS (ESI) calc for $\mathrm{C}_{17} \mathrm{H}_{28} \mathrm{O}_{5} \mathrm{NNaPS}[\mathrm{M}+\mathrm{Na}]^{+}$412.13180; found: 412.13169 .

[(R,S)-1-Amino-3-(phenylsulfanyl)propyl]phosphonic acid (10a). From 2a: Compound 2a (13 g; $24.3 \mathrm{mmol}$ ) was heated for $12 \mathrm{~h}$ at reflux with $50 \mathrm{ml}$ of $35 \% \mathrm{HCl}$ and $50 \mathrm{ml}$ of glacial acetic acid. The solvents were evaporated under reduced pressure, and the resultant oily residue was taken up in $50 \mathrm{ml}$ of methanol. The methanolic solution of the crude product was treated with methyloxirane until a $\mathrm{pH}$ of 6 was reached. The product precipitated, was removed by filtration and then washed with methanol and diethyl ether. The yield was $4 \mathrm{~g}$ (67\%). The product was a white solid, $\mathrm{mp} 266-268^{\circ} \mathrm{C}$.

From 8a: Compound 8a (0.6 g; $1.5 \mathrm{mmol})$ was stirred for $24 \mathrm{~h}$ in $2 \mathrm{ml}$ of $35 \%$ of $\mathrm{HBr} / \mathrm{AcOH}$. The solvent was evaporated at $60^{\circ} \mathrm{C}$ in vacuo, and the residue was treated with 1,2-epoxypropane in methanol as described above. The yield was $0.25 \mathrm{~g}(69 \%)$.

From 7a: Compound 7a $(0.3 \mathrm{~g} ; 0.65 \mathrm{mmol})$ was dissolved in $5 \mathrm{ml}$ of a mixture TFA-DCMDMS- $\mathrm{H}_{2} \mathrm{O}(47.5: 47.5: 2.5: 2.5)$, and the solution was stirred at rt overnight. The volatile liquids (DCM and DMS) were removed by rotary evaporator at $25^{\circ} \mathrm{C}$, and the remaining yellow solution (approximately $2 \mathrm{ml}$ ) was lyophilized twice with $5 \mathrm{ml}$ of water. The residue was dissolved in $10 \mathrm{ml}$ of $0.1 \%$ aqueous TFA, passed through a filter (Rotilabo $0.22 \mu \mathrm{m}$, the compound tends to precipitate from the solution) and subjected to preparative RP-HPLC (G1). Finally, the pure product was lyophilized from water. The yield was $113 \mathrm{mg}(73 \%)$. The product was a white powder.

From 9a: $\operatorname{TMSBr}(1.5 \mathrm{~g} ; 10 \mathrm{mmol})$ was added to the solution of $9 \mathbf{a}(0.4 \mathrm{~g} ; 1 \mathrm{mmol})$ in $10 \mathrm{ml}$ of anhydrous acetonitrile, and the reaction was allowed to proceed at $\mathrm{rt}$ for 48 hours excluded from air and moisture. The reaction was then concentrated under reduced pressure; the residue was dissolved in $5 \mathrm{ml}$ of a mixture TFA-DCM-DMS- $\mathrm{H}_{2} \mathrm{O}(47.5: 47.5: 2.5: 2.5)$ and stirred at $\mathrm{rt}$ overnight. The isolation of target product 10a was performed in the same fashion as described for the reaction from $\mathbf{7 a}$ to $\mathbf{1 0 a}$. Yield $130 \mathrm{mg}(50 \%)$. White powder. $\mathrm{R}_{\mathrm{f}}=0.35$ (S5). Calcd. for $\mathrm{C}_{9} \mathrm{H}_{14} \mathrm{NO}_{3} \mathrm{PS}$ (247.3) C $43.72 \%$, H 5.71\%, N 5.66 \%. Found: C 43.47\%, H 5.62\%, N 5.52\%. ${ }^{1} \mathrm{H}$ NMR (600 MHz; D $2 \mathrm{O}+\mathrm{NaOD}): \delta_{\mathrm{H}} 1.72(1 \mathrm{H}, \mathrm{m}, \mathrm{C}-\mathrm{C} \underline{\mathrm{HaHb}}-\mathrm{C}), 2.10$ (1H, m, C-CHaHb-C), 2.69 $\left(1 \mathrm{H}, \mathrm{m}, \mathrm{dtd},{ }^{3} J_{\mathrm{HP}}=11.4,{ }^{3} J_{\mathrm{HH}}=10.5\right.$ and $\left.3.4 \mathrm{~Hz}, \mathrm{~N}-\mathrm{CH}-\mathrm{P}\right), 3.06\left(1 \mathrm{H}, \mathrm{ddd},{ }^{2} J_{\mathrm{HH}}=13.0,{ }^{3} J_{\mathrm{HH}}=\right.$ 9.2 and $7.0 \mathrm{~Hz}, \mathrm{~S}-\mathrm{C} \underline{\mathrm{HaHb}}), 3.26\left(1 \mathrm{H}, \mathrm{ddd},{ }^{2} J_{\mathrm{HH}}=13.0,{ }^{3} J_{\mathrm{HH}}=9.6\right.$ and $\left.4.7 \mathrm{~Hz}, \mathrm{~S}-\mathrm{C} \underline{\mathrm{HaHb}}\right), 7.29$ $\left(1 \mathrm{H}_{\text {arom }}, \mathrm{m}, 1 \mathrm{CH}\right), 7.40\left(2 \mathrm{H}_{\text {arom }}, \mathrm{m}, 2 \mathrm{CH}\right), 7.46\left(2 \mathrm{H}_{\text {arom }}, \mathrm{m}, 2 \mathrm{CH}\right) .{ }^{13} \mathrm{C} \mathrm{NMR}\left(150.9 \mathrm{MHz} ; \mathrm{D}_{2} \mathrm{O}+\right.$ $\mathrm{NaOD}): \delta_{\mathrm{C}} 33.41\left(1 \mathrm{C}, \mathrm{d},{ }^{3} J_{\mathrm{CP}}=14.5 \mathrm{~Hz}, \mathrm{~S}-\mathrm{CH}_{2}\right), 34.20\left(1 \mathrm{C}, \mathrm{C}-\underline{C H}_{2}-\mathrm{C}\right), 52.14\left(1 \mathrm{C}, \mathrm{d},{ }^{1} J_{\mathrm{CP}}=\right.$ 
137.7 Hz, N-CH-P), 128.94 (1C, para-CHarom), 131.39 (2C, ortho- $\left.\mathrm{CH}_{\text {arom }}\right), 132.05$ (2C, meta-

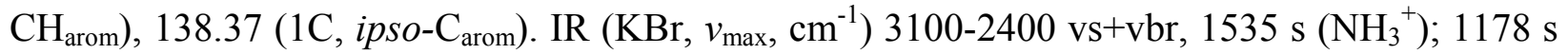
$\left(\mathrm{PO}_{2}{ }^{-}\right) ; 1023$ vs $(\mathrm{POH}) ; 1585 \mathrm{~m}, 1481 \mathrm{~m}, 1439 \mathrm{~m}, 736 \mathrm{~s}, 689 \mathrm{~s}$ (ring). HRMS (ESI) calc for $\mathrm{C}_{9} \mathrm{H}_{13} \mathrm{O}_{3}$ NPS [M-1] ${ }^{+}$246.03592; found: 246.03588 .

[(R,S)-1-Amino-3-(tert-butylsulfanyl)propyl]phosphonic acid (10b). From 8b: The acid 10b was prepared in the same fashion as $\mathbf{1 0 a}$ by the reaction of $\mathbf{8 b}(0.6 \mathrm{~g} ; 1.4 \mathrm{mmol})$ and $2 \mathrm{ml}$ of $35 \%$ $\mathrm{HBr} / \mathrm{AcOH}$. The precipitated oil was separated and subjected to RP-HPLC. Only traces of the product were isolated.

From 7b: The acid $\mathbf{1 0 b}$ was prepared in the same fashion as $\mathbf{1 0 a}$ by the treatment of $\mathbf{7 b}(0.5 \mathrm{~g}$; $1.1 \mathrm{mmol}$ ) with $5 \mathrm{ml}$ of the cleavage cocktail. RP-HPLC (G2). The yield was $168 \mathrm{mg}(68 \%)$. The product was a white powder.

From $9 \mathbf{b}$ : The acid $\mathbf{1 0 b}$ was prepared in the same fashion as $\mathbf{1 0 a}$ by the reaction of $\mathbf{9 b}(0.6 \mathrm{~g} ; 1.7$ $\mathrm{mmol})$ and TMSBr $(2.6 \mathrm{~g} ; 17 \mathrm{mmol})$ followed by the treatment of the resulting oil with $5 \mathrm{ml}$ of the cleavage cocktail. Yield $195 \mathrm{mg}(51 \%)$. White powder. $\mathrm{R}_{\mathrm{f}}=0.29$ (S5). Calcd. for $\mathrm{C}_{7} \mathrm{H}_{18} \mathrm{NO}_{3} \mathrm{PS}$ (227.3) C $37.00 \%$, H 7.98\%, N 6.16 \%. Found: C 36.76\%, H 7.87\%, N 5.81\%. ${ }^{1} \mathrm{H}$ NMR (600 MHz; D $2 \mathrm{O}+\mathrm{NaOD}): \delta_{\mathrm{H}} 1.33\left(9 \mathrm{H}, \mathrm{s}, \mathrm{C}\left(\mathrm{CH}_{3}\right)_{3}\right), 1.59(1 \mathrm{H}, \mathrm{m}, \mathrm{C}-\mathrm{CHaHb}-\mathrm{C}), 1.97(1 \mathrm{H}$, $\mathrm{m}, \mathrm{C}-\mathrm{CHaHb}-\mathrm{C}), 2.58\left(1 \mathrm{H}, \mathrm{m}, \mathrm{td},{ }^{3} J_{\mathrm{HP}}=11.0,{ }^{3} J_{\mathrm{HH}}=11.0\right.$ and $\left.3.1 \mathrm{~Hz}, \mathrm{~N}-\mathrm{CH}-\mathrm{P}\right), 2.65(1 \mathrm{H}$, ddd, ${ }^{2} J_{\mathrm{HH}}=11.8,{ }^{3} J_{\mathrm{HH}}=9.8$ and $\left.6.8 \mathrm{~Hz}, \mathrm{~S}-\mathrm{CHaHb}\right), 2.85\left(1 \mathrm{H}, \mathrm{ddd},{ }^{2} J_{\mathrm{HH}}=11.8,{ }^{3} J_{\mathrm{HH}}=10.2\right.$ and 4.8 $\mathrm{Hz}, \mathrm{S}-\mathrm{CHaHb}) .{ }^{13} \mathrm{C} \mathrm{NMR}\left(150.9 \mathrm{MHz} ; \mathrm{D}_{2} \mathrm{O}+\mathrm{NaOD}\right): \delta_{\mathrm{C}} 28.64\left(1 \mathrm{C}, \mathrm{d},{ }^{3} J_{\mathrm{CP}}=14.5 \mathrm{~Hz}, \mathrm{~S}-\mathrm{CH}_{2}\right)$, $32.88\left(3 \mathrm{C}, \mathrm{C}\left(\underline{\mathrm{CH}}_{3}\right)_{3}\right), 34.99\left(1 \mathrm{C}, \underline{\mathrm{C}}\left(\mathrm{CH}_{3}\right)_{3}\right), 45.32\left(1 \mathrm{C}, \mathrm{C}-\underline{C H}_{2}-\mathrm{C}\right), 52.76\left(1 \mathrm{C}, \mathrm{d},{ }^{1} J_{\mathrm{CP}}=137.6 \mathrm{~Hz}\right.$, $\mathrm{N}-\mathrm{CH}-\mathrm{P})$. IR (KBr, $\left.v_{\max }, \mathrm{cm}^{-1}\right) \quad 3100-2400 \mathrm{vs}+\mathrm{vbr}, 1535 \mathrm{~s}\left(\mathrm{NH}_{3}{ }^{+}\right) ; 1170$ vs $\left(\mathrm{PO}_{2}^{-}\right) ; 1027$ vs (POH); 2962 vs $\left(\mathrm{CH}_{3}\right)$. HRMS (ESI) calc for $\mathrm{C}_{7} \mathrm{H}_{18} \mathrm{O}_{3} \mathrm{NPS}$ [M-1] $]^{+} 226.06722$; found: 226.06730 .

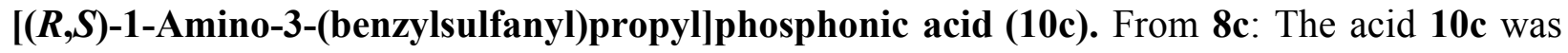
prepared in the same fashion as $\mathbf{1 0 a}$ by the reaction of $8 \mathrm{c}(0.6 \mathrm{~g} ; 1.4 \mathrm{mmol})$ and $2 \mathrm{ml}$ of $35 \%$ $\mathrm{HBr} / \mathrm{AcOH}$. The yield was $0.23 \mathrm{~g}(62 \%)$. The product was a white solid, mp $264-266{ }^{\circ} \mathrm{C}$ (lit. ${ }^{7}$ $\left.270-272^{\circ} \mathrm{C}\right)$.

From 7c: The acid 10c was prepared in the same fashion as $10 a$ by the treatment of $7 \mathbf{c}(0.5 \mathrm{~g} ; 1$ mmol) with $5 \mathrm{ml}$ of the cleavage cocktail. RP-HPLC (G2). The yield was $200 \mathrm{mg}$ (75\%). The product was a white powder.

From 9c: The acid 10c was prepared in the same fashion as $10 a$ by the reaction of $9 \mathrm{c}(0.4 \mathrm{~g} ; 1$ mmol) and TMSBr $(1.5 \mathrm{~g} ; 10 \mathrm{mmol})$ followed by the treatment of the residual oil with $5 \mathrm{ml}$ of the cleavage cocktail. Yield $147 \mathrm{mg}(55 \%)$. White powder. $\mathrm{R}_{\mathrm{f}}=0.33$ (S5). Calcd. for $\mathrm{C}_{10} \mathrm{H}_{16} \mathrm{NO}_{3} \mathrm{PS}$ (261.3) C $45.97 \%$, H 6.17\%, N $5.36 \%$. Found: C 45.57\%, H 6.02\%, N 5.26\%. ${ }^{1} \mathrm{H}$ NMR (600 MHz; D $2 \mathrm{O}+\mathrm{NaOD}): \delta_{\mathrm{H}} 1.64(1 \mathrm{H}, \mathrm{m}, \mathrm{C}-\mathrm{C} \underline{\mathrm{HaHb}}-\mathrm{C}), 2.07$ (1H, m, C-CHaHb-C), 2.60 $\left(1 \mathrm{H}, \mathrm{m}, \mathrm{ddd},{ }^{3} J_{\mathrm{HP}}=11.2,{ }^{3} J_{\mathrm{HH}}=10.6\right.$ and $\left.3.1 \mathrm{~Hz}, \mathrm{~N}-\mathrm{CH}-\mathrm{P}\right), 2.55\left(1 \mathrm{H}, \mathrm{ddd},{ }^{2} J_{\mathrm{HH}}=12.8,{ }^{3} J_{\mathrm{HH}}=\right.$ 9.4 and $7.0 \mathrm{~Hz}, \mathrm{~S}-\mathrm{C} \underline{\mathrm{HaHb}}), 2.73\left(1 \mathrm{H}, \mathrm{ddd},{ }^{2} J_{\mathrm{HH}}=12.8,{ }^{3} J_{\mathrm{HH}}=9.7\right.$ and $\left.4.6 \mathrm{~Hz}, \mathrm{~S}-\mathrm{C} \underline{\mathrm{HaHb}}\right), 3.81$ $\left(2 \mathrm{H}, \mathrm{s}, \mathrm{S}-\mathrm{CH}_{2}\right), 7.35\left(1 \mathrm{H}_{\text {arom }}, \mathrm{m}, 1 \mathrm{CH}\right), 7.39\left(4 \mathrm{H}_{\text {arom }}, \mathrm{m}, 4 \mathrm{CH}\right) .{ }^{13} \mathrm{C} \mathrm{NMR}\left(150.9 \mathrm{MHz} ; \mathrm{D}_{2} \mathrm{O}+\right.$ $\mathrm{NaOD}): \delta_{\mathrm{C}} 31.45\left(1 \mathrm{C}, \mathrm{d},{ }^{3} J_{\mathrm{CP}}=14.7 \mathrm{~Hz}, \mathrm{~S}-\mathrm{CH}_{2}\right), 34.12\left(1 \mathrm{C}, \mathrm{S}-\mathrm{CH}_{2}\right), 37.63\left(1 \mathrm{C}, \mathrm{C}-\underline{C H}_{2}-\mathrm{C}\right)$, $52.17\left(1 \mathrm{C}, \mathrm{d},{ }^{1} J_{\mathrm{CP}}=137.9 \mathrm{~Hz}, \mathrm{~N}-\mathrm{CH}-\mathrm{P}\right), 129.94\left(1 \mathrm{C}\right.$, para- $\left.\mathrm{CH}_{\text {arom }}\right), 131.56$ (2C, ortho- $\left.\mathrm{CH}_{\text {arom }}\right)$, 131.70 (2C, meta- $\left.\mathrm{CH}_{\text {arom }}\right), 141.43$ (1C, ipso- $\left.\mathrm{C}_{\text {arom }}\right)$. IR (KBr, $\left.v_{\max }, \mathrm{cm}^{-1}\right) 3200-2400 \mathrm{vs}+\mathrm{vbr}, 1535$ 
s $\left(\mathrm{NH}_{3}{ }^{+}\right) ; 1177 \mathrm{~s}\left(\mathrm{PO}_{2}^{-}\right) ; 1023$ vs $(\mathrm{POH}) ; 1494 \mathrm{~m}, 1463 \mathrm{~m}, 697 \mathrm{~m}$ (ring). HRMS (ESI) calc for $\mathrm{C}_{10} \mathrm{H}_{15} \mathrm{O}_{3} \mathrm{NPS}[\mathrm{M}-1]^{+}$260.05157; found: 260.05124 .

\section{Acknowledgements}

This work was supported by the Grant Agency of the Czech Republic (projects P207/10/1277 to JJ and P203/09/1919 to MB) and by the Research Project of the Academy of Sciences of the Czech Republic (Z40550506, to IOCB).

\section{References}

1. Collinsova, M.; Castro, C.; Garrow, T. A.; Yiotakis, A.; Dive, V.; Jiracek, J. Chem. Biol. 2003, 10, 113-122.

2. Jiracek, J.; Collinsova, M.; Rosenberg, I.; Budesinsky, M.; Protivinska, E.; Netusilova, H.; Garrow, T. A. J. Med. Chem. 2006, 49, 3982-3989.

3. Vanek, V.; Budesinsky, M.; Kabeleova, P.; Sanda, M.; Kozisek, M.; Hanclova, I.; Mladkova, J.; Brynda, J.; Rosenberg, I.; Koutmos, M.; Garrow, T. A.; Jiracek, J. J. Med. Chem. 2009, 52, 3652-3665.

4. Szegedi, S. S.; Castro, C.; Koutmos, M.; Garrow, T. A. J. Biol. Chem. 2008, 283, 89398945.

5. Mucha, A.; Kafarski, P.; Berlicki, L. J. Med. Chem. 2011, 54, 5955-5980.

6. Collinsova, M.; Jiracek, J. Curr. Med. Chem. 2000, 7, 629-647.

7. Kudzin, Z. H.; Stec, W. J. Synthesis-Stuttgart 1980, 1032-1034.

8. Kudzin, Z. H.; Mokrzan, J.; Skowronski, R. Phosphorus Sulfur Silicon Relat. Elem. 1989, 42, 41-46.

9. Tam, C. C.; Mattocks, K. L.; Tishler, M. Synthesis-Stuttgart 1982, 188-190.

10. Zefirov, N. S.; Matveeva, E. D. Arkivoc 2008, 1-17.

11. Oleksyszyn, J.; Tyka, R. Tetrahedron Lett. 1977, 2823-2824.

12. Oleksyszyn, J.; Subotkowska, L.; Mastalerz, P. Synthesis-Stuttgart 1979, 985-986.

13. Kudzin, Z. H.; Stec, W. J. Synthesis-Stuttgart 1983, 812-814.

14. Kudzin, M. H.; Kudzin, Z. H.; Drabowicz, J. Arkivoc 2011, 227-269.

15. Van der Veken, P.; El Sayed, I.; Joossens, J.; Stevens, C. V.; Augustyns, K.; Haemers, A. Synthesis-Stuttgart 2005, 634-638.

16. Yuan, C. Y.; Wang, G. H.; Chen, S. J. Synthesis-Stuttgart 1990, 522-524.

17. Montzka, T. A. U.S. Patent 3378 561, 1968.

18. Vinton, W. H. U.S. Patent 2461 013, 1949.

19. Vo-Quang, Y.; Carniato, D.; Liliane, V. Q.; Lacoste, A. M.; Neuzil, E.; Legoffic, F. J. Med. Chem. 1986, 29, 579-581.

20. Tsuzuki, Y.; Chiba, K.; Mizuno, K.; Tomita, K.; Suzuki, K. Tetrahedron-Asymmetry 2001, 12, 2989-2997. 